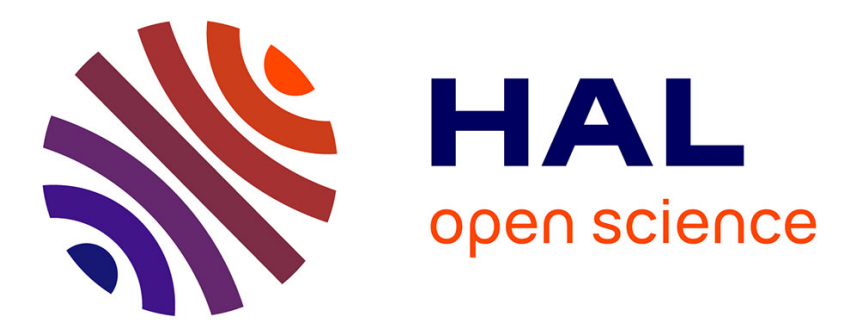

\title{
A stabilized Lagrange multiplier method for the enriched finite-element approximation of Tresca contact problems of cracked elastic bodies
}

Saber Amdouni, Maher Moakher, Yves Renard

\section{- To cite this version:}

Saber Amdouni, Maher Moakher, Yves Renard. A stabilized Lagrange multiplier method for the enriched finite-element approximation of Tresca contact problems of cracked elastic bodies. 2013. hal-00845770

\author{
HAL Id: hal-00845770 \\ https://hal.science/hal-00845770
}

Preprint submitted on 18 Jul 2013

HAL is a multi-disciplinary open access archive for the deposit and dissemination of scientific research documents, whether they are published or not. The documents may come from teaching and research institutions in France or abroad, or from public or private research centers.
L'archive ouverte pluridisciplinaire HAL, est destinée au dépôt et à la diffusion de documents scientifiques de niveau recherche, publiés ou non, émanant des établissements d'enseignement et de recherche français ou étrangers, des laboratoires publics ou privés. 


\title{
A stabilized Lagrange multiplier method for the enriched finite-element approximation of Tresca contact problems of cracked elastic bodies
}

\author{
S. Amdouni ${ }^{1}$, M. Moakher ${ }^{2}$, Y. Renard $^{3}$
}

\begin{abstract}
In this paper we propose a local projection stabilized Lagrange multiplier method in order to approximate the two-dimensional linear elastostatics unilateral contact problem with Tresca friction in the framework of the eXtended Finite Element Method X-FEM. This last method allows to perform finite-element computations on cracked domains by using meshes of the non-cracked domain. The advantage of the used stabilization technique is to affect only the equation on multipliers and thus to be equation independent. We study the existence, uniqueness and a priori error estimate of three hybrid discrete formulations.
\end{abstract}

\section{Introduction}

The use of Lagrange multiplier methods to formulate contact problems is a well-known and powerful technique. One difficulty, especialy for fictitious domain like method, is due to the fact that a discrete compatibility condition between the finite-element space for the displacement and the one for the multiplier is required, namely the uniform discrete L.B.B. or inf-sup condition. To circumvent this difficulty some stabilization techniques have been developed. One of the most used technique is the Barbosa-Hughes stabilization $[7,8]$ where the stability is provided by adding a supplementary term involving an approximation of the normal derivative of the primal variable on $\Gamma_{C}$ (see [20] and [3]). This method of stabilization depends on the problem to be solved which makes its implementation a difficult task. In this study we will focus on local projection stabilization techniques where the difference of the multiplier with its projection on some inf-sup stable spaces is penalized to ensure the stability of the approximation problem. This stabilized technique is asymptotically consistent and affects only the multiplier equations in a manner that is independent of the problem to be solved. To the best of our knowledge, this technique was used for the first time by Silvester [29] in the context of incompressible flow. A theoretical framework was developed in Brezzi et al. [11] for mixed finite-element methods and by Burman [13] for Lagrange multiplier method to prescribe some constraints on the interface. Recently, this technique was proposed and analyzed in the framework of finite-element method with a fictitious domain approach in $[5,9]$.

The purpose of this contribution is to adapt the local projection stabilization technique to the enriched finite-element approximation of contact problems with Tresca friction of cracked elastic bodies and to provide a priori error estimates of the stabilized formulation. Let us remark that a priori error estimates we propose are not optimal. However, the existing analysis of the approximation of Tresca contact problem even for standard approximations and especially for Lagrange multiplier methods are also suboptimal. Let us recall the main existing result

\footnotetext{
${ }^{1}$ Laboratoire LAMSIN, Ecole Nationale d'Ingénieurs de Tunis, Université Tunis El Manar, B.P. 37, 1002 Tunis-Belvédère, Tunisie \& INSA-Lyon, ICJ UMR5208-France. Saber.Amdouni@insa-lyon.fr Phone: +33 472438287

${ }^{2}$ Laboratoire LAMSIN, Ecole Nationale d'Ingénieurs de Tunis, Université Tunis El Manar, B.P. 37, 1002 Tunis-Belvédère, Tunisie. maher.moakher@enit.rnu.tn Phone: +21671874700 (ext.559),

${ }^{3}$ Université de Lyon, CNRS, INSA-Lyon, ICJ UMR5208, LaMCoS UMR5259, F-69621, Villeurbanne, France. Yves.Renard@insa-lyon.fr Phone: +33472438708, Fax: +33472438529
} 
for standard approximations. An order of $h^{3 / 4}$ is proved using continuous piecewise linear normal multiplier and weakly continuous piecewise linear tangent multiplier [6] and an order of $h \sqrt[4]{|\log (h)|}$ is proved with the additional assumptions that we have a finite number of transitions between contact and non-contact zones, the jump of the displacement on $\Gamma_{C}$ is in $W^{1, \infty}$, the tangent stress $\lambda_{t} \in L^{\infty}\left(\Gamma_{C}\right)$ and the given slip $s \in L^{\infty}\left(\Gamma_{C}\right)$. In the same context of [6] an order of $h^{1 / 2}$ is proved using piecewise constant shape functions (see [28] and [21]). This estimate can be improved (a convergence rate of order $h^{3 / 4}$ ) under the additional assumption that the slip bound $s$ is a positive constant on $\Gamma_{C}$ (see [28] and [21]).

The paper is organized as follows. In Section 2, we introduce the formulation of the unilateral contact problem with Tresca friction on a crack of an elastic structure. In Section 3, we present the elasticity problem approximated by both the enrichment strategy introduced in [14] and the local projection stabilized Lagrange multiplier method [5]. We show the existence and uniqueness of the solution of the stabilized formulation. Also we prove a priori error estimates following three different discrete contact conditions (the study is restricted to piecewise affine and constant finite element methods). Finally, in Section 4, we present some numerical experiments on a very simple situation. We compare the stabilized and the non-stabilized cases for different finite-element approximations. The influence of the stabilization parameters is also investigated.

\section{Formulation of the continuous problem}

We start by introducing some useful notations and several functional spaces. In what follows, bold lowercase letters like $\mathbf{u}, \mathbf{v}$, indicate vector-valued quantities, while the bold capital ones (e.g., $\mathbf{V}, \mathbf{K}, \ldots$ ) represent functional sets involving vector fields. As usual, we denote by $\left(L^{2}(.)\right)^{d}$ and by $\left(H^{s}(.)\right)^{d}, s \geq 0, d=1,2$ the classical Lebesgue and Hilbert spaces in $d$-dimensional space (see [1]). The usual norm of $\left(H^{s}(D)\right)^{d}$ is denoted by $\|\cdot\|_{s, D}$ and we keep the same notation when $d=1$ or $d=2$. For shortness, the $\left(L^{2}(D)\right)^{d}$-norm will be denoted by $\|\cdot\|_{0, D}$ when $d=1$ or $d=2$. In the sequel the symbol $|\cdot|$ will denote either the Euclidean norm in $\mathbb{R}^{2}$, the length of a line segment, or the area of a planar domain.

Let us consider the deformation of a cracked elastic body occupying, in the initial configuration, a domain $\Omega$ in $\mathbb{R}^{2}$ where plane small strains are assumed. The boundary $\partial \Omega$ of the domain $\Omega$ is assumed to be polygonal (for simplicity) and consists of three non-overlapping parts $\Gamma_{D}$, $\Gamma_{N}$ and $\Gamma_{C}$ with meas $\left(\Gamma_{D}\right)>0$ and meas $\left(\Gamma_{C}\right)>0$. The body is clamped on $\Gamma_{D}$. It is subjected to volume forces $\mathbf{f}=\left(f_{1}, f_{2}\right) \in\left(L^{2}(\Omega)\right)^{2}$ and to surface loads $\mathbf{g}=\left(g_{1}, g_{2}\right) \in\left(L^{2}\left(\Gamma_{N}\right)\right)^{2}$. The boundary part $\Gamma_{C}$ (or the crack location) is supposed to be a straight line segment. We denote by $\Gamma_{C+}$ and $\Gamma_{C-}$ each of the two sides of the crack (see Fig. 1). We suppose that we have a frictional contact condition between $\Gamma_{C+}$ and $\Gamma_{C-}$. Of course, in the initial configuration, both $\Gamma_{C+}$ and $\Gamma_{C-}$ coincide. Let $\mathbf{n}=\mathbf{n}^{+}=-\mathbf{n}^{-}=\left(n_{1}, n_{2}\right)$ denote the outward normal unit vector on $\Gamma_{C+}$ and $\mathbf{t}=\mathbf{t}^{+}=-\mathbf{t}^{-}=\left(-n_{2}, n_{1}\right)$ an associated unit tangent vector. 


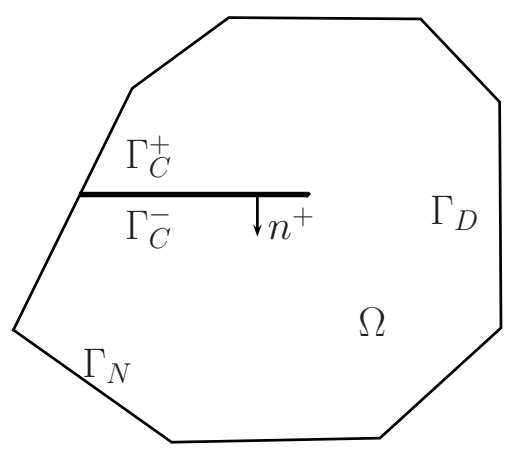

Figure 1: A cracked domain.

Under plane small strain assumptions, the problem of homogeneous isotropic linear elasticity consists in finding the displacement field $\mathbf{u}: \Omega \rightarrow \mathbb{R}^{2}$ satisfying

$$
\begin{aligned}
\operatorname{div} \boldsymbol{\sigma}(\mathbf{u})+\mathbf{f} & =\mathbf{0} & & \text { in } \Omega, \\
\boldsymbol{\sigma}(\mathbf{u}) & =\lambda_{L} \operatorname{tr} \varepsilon(\mathbf{u}) I+2 \mu_{L} \varepsilon(\mathbf{u}), & & \text { in } \Omega, \\
\mathbf{u} & =\mathbf{0} & & \text { on } \Gamma_{D}, \\
\boldsymbol{\sigma}(\mathbf{u}) \mathbf{n} & =\mathbf{g} & & \text { on } \Gamma_{N}
\end{aligned}
$$

where $\boldsymbol{\sigma}=\left(\sigma_{i j}\right), 1 \leq i, j \leq 2$, stands for the stress tensor field, $\boldsymbol{\varepsilon}(\mathbf{v})=\left(\boldsymbol{\nabla} \mathbf{v}+\boldsymbol{\nabla} \mathbf{v}^{T}\right) / 2$ represents the linearized plane strain tensor field, $\lambda_{L} \geq 0, \mu_{L}>0$ are the Lamé coefficients, and $I$ denotes the identity tensor. For a displacement field $\mathbf{v}$ and a density of surface forces $\boldsymbol{\sigma}(\mathbf{v}) \mathbf{n}$ defined on $\partial \Omega$, we adopt the following notations:

$$
\mathbf{v}^{+}=v_{n}^{+} \mathbf{n}^{+}+v_{t}^{+} \mathbf{t}^{+}, \quad \mathbf{v}^{-}=v_{n}^{-} \mathbf{n}^{-}+v_{t}^{-} \mathbf{t}^{-} \quad \text { and } \quad \boldsymbol{\sigma}(\mathbf{v}) \mathbf{n}=\sigma_{n}(\mathbf{v}) \mathbf{n}+\sigma_{t}(\mathbf{v}) \mathbf{t},
$$

where $\mathbf{v}^{+}$(resp. $\mathbf{v}^{-}$) is the trace of displacement on $\Gamma_{C}$ on the $\Gamma_{C}^{+}$side (resp. on the $\Gamma_{C}^{-}$side). The conditions describing the unilateral contact on $\Gamma_{C}$ are:

$$
\llbracket u_{n} \rrbracket=u_{n}^{+}+u_{n}^{-} \leq 0, \quad \sigma_{n}(\mathbf{u}) \leq 0, \quad \sigma_{n}(\mathbf{u}) \cdot \llbracket u_{n} \rrbracket=0,
$$

where $\llbracket u_{n} \rrbracket$ is the jump of the normal displacement across the crack $\Gamma_{C}$. Let us denote by $s \geq 0$ the given friction threshold on $\Gamma_{C}$ (which is assumed to be constant for the sake simplicity). The static Tresca friction condition reads as follows:

$$
\begin{cases}\left|\sigma_{t}(\mathbf{u})\right| \leq s, & \text { a.e. on } \Gamma_{C}, \\ \text { if } \quad\left|\sigma_{t}(\mathbf{u})\right|<s, & \text { then } \llbracket u_{t} \rrbracket=0, \\ \text { if } \quad\left|\sigma_{t}(\mathbf{u})\right|=s, & \text { then there exists } \nu \geq 0 \text { such that } \llbracket u_{t} \rrbracket=-\nu \sigma_{t}(\mathbf{u}),\end{cases}
$$

with $\llbracket \mathbf{u}_{t} \rrbracket=u_{t}^{+}+u_{t}^{-}$. In the classical weak formulation of Problem (1)-(6), we need to use the following Hilbert spaces:

$$
\begin{aligned}
\mathbf{V} & \left.=\left\{\mathbf{v} \in\left(H^{1}(\Omega)\right)\right)^{2}: \quad \mathbf{v}=\mathbf{0} \text { on } \Gamma_{D}\right\}, \quad \mathbf{W}=W_{N} \times W_{T}, \\
W_{N} & =\left\{f_{n} \in L^{2}\left(\Gamma_{C}\right): \exists \mathbf{v} \in \mathbf{V} \text { such that } f_{n}=\llbracket v_{n} \rrbracket\right\}, \\
W_{T} & =\left\{f_{t} \in L^{2}\left(\Gamma_{C}\right): \exists \mathbf{v} \in \mathbf{V} \text { such that } f_{t}=\llbracket v_{t} \rrbracket\right\},
\end{aligned}
$$


and their topological dual spaces $\mathbf{V}^{\prime}, W_{N}^{\prime}$ and $W_{T}^{\prime}$, endowed with the following norms:

$$
\begin{aligned}
\|\mathbf{v}\| & =(a(\mathbf{v}, \mathbf{v}))^{1 / 2}, \quad\|\mathbf{f}\|_{\mathbf{w}}=\left(\left\|f_{n}\right\|_{W_{N}}^{2}+\left\|f_{t}\right\|_{W_{T}}^{2}\right)^{1 / 2}, \\
\left\|f_{n}\right\|_{W_{N}} & =\inf _{\mathbf{v} \in \mathbf{V} ; f_{n}=\llbracket v_{n} \rrbracket}\|\mathbf{v}\|, \quad\left\|f_{t}\right\|_{W_{T}}=\inf _{\mathbf{v} \in \mathbf{V} ; f_{t}=\llbracket v_{t} \rrbracket}\|\mathbf{v}\|, \\
\left\|\mu_{n}\right\|_{W_{N}^{\prime}} & =\sup _{\mathbf{v} \in \mathbf{V}} \frac{\left\langle\mu_{n}, \llbracket v_{n} \rrbracket\right\rangle_{W_{N}^{\prime}, W_{N}}}{\|\mathbf{v}\|}, \quad\left\|\mu_{t}\right\|_{W_{T}^{\prime}}=\sup _{\mathbf{v} \in \mathbf{V}} \frac{\left\langle\mu_{t}, \llbracket v_{t} \rrbracket\right\rangle_{W_{T}^{\prime}, W_{T}}}{\|\mathbf{v}\|} .
\end{aligned}
$$

With the following norms we prove easily that:

$$
\begin{aligned}
\left\|v_{n}\right\|_{W_{N}} & \leq\|\mathbf{v}\|_{\mathbf{v}}, \forall \mathbf{v} \in \mathbf{V}, \\
\left\|v_{t}\right\|_{W_{T}} & \leq\|\mathbf{v}\|_{\mathbf{v}}, \forall \mathbf{v} \in \mathbf{V}, \\
c_{1}\left\|\mu_{n}\right\|_{W_{N}^{\prime}} & \leq\left\|\mu_{n}\right\|_{0, \Gamma_{C}} \forall \mu_{n} \in W_{N}^{\prime}, \\
c_{1}\left\|\mu_{t}\right\|_{W_{T}^{\prime}} & \leq\left\|\mu_{t}\right\|_{0, \Gamma_{C}} \forall \mu_{t} \in W_{N}^{\prime},
\end{aligned}
$$

where $c_{1}>0$ is the inverse of the trace constant. Next we define the convex set of admissible Lagrange multipliers denoted:

$$
\begin{gathered}
\mathbf{M}(s)=M_{N} \times M_{T}(s), \\
M_{N}=\left\{\mu_{n} \in W_{N}^{\prime}:\left\langle\mu_{n}, f_{n}\right\rangle_{W_{N}^{\prime}, W_{N}} \geq 0 \text { for all } f_{n} \in W_{N}, f_{n} \leq 0 \text { a.e. on } \Gamma_{C}\right\}, \\
M_{T}(s)=\left\{\mu_{t} \in W_{T}^{\prime}:\left\langle\mu_{t}, f_{t}\right\rangle_{W_{T}^{\prime}, W_{T}}+\left\langle s,\left|f_{t}\right|\right\rangle_{W_{T}^{\prime}, W_{T}} \geq 0 \text { for all } f_{t} \in W_{T}\right\},
\end{gathered}
$$

where the notation $\langle\cdot, \cdot\rangle_{W_{N}^{\prime}, W_{N}}$ (resp. $\langle\cdot, \cdot\rangle_{W_{T}^{\prime}, W_{T}}$ ) stands for the duality pairing between $W_{N}^{\prime}$ and $W_{N}$ (resp. between $W_{T}^{\prime}$ and $W_{T}$ ). The mixed formulation of the Tresca contact problem (1)-(6) consists then in finding $\mathbf{u} \in \mathbf{V}$ and $\boldsymbol{\lambda} \in \mathbf{M}(s)$ such that

$$
\begin{cases}a(\mathbf{u}, \mathbf{v})-b(\boldsymbol{\lambda}, \mathbf{v})=L(\mathbf{v}), & \forall \mathbf{v} \in \mathbf{V}, \\ b(\boldsymbol{\mu}-\boldsymbol{\lambda}, \mathbf{u}) \geq 0, & \forall \boldsymbol{\mu} \in \mathbf{M}(s),\end{cases}
$$

where

$$
\begin{aligned}
a(\mathbf{u}, \mathbf{v}) & =\int_{\Omega} \boldsymbol{\sigma}(\mathbf{u}): \boldsymbol{\varepsilon}(\mathbf{v}) d \Omega, \\
L(\mathbf{v}) & =\int_{\Omega} \mathbf{f} \cdot \mathbf{v} d \Omega+\int_{\Gamma_{N}} \mathbf{g} \cdot \mathbf{v} d \Gamma .
\end{aligned}
$$

An equivalent formulation of (7) consists in finding $(\mathbf{u}, \boldsymbol{\lambda}) \in \mathbf{V} \times \mathbf{M}(s)$ satisfying

$$
\mathscr{L}(\mathbf{u}, \boldsymbol{\mu}) \leq \mathscr{L}(\mathbf{u}, \boldsymbol{\lambda}) \leq \mathscr{L}(\mathbf{v}, \boldsymbol{\lambda}), \quad \forall \mathbf{v} \in \mathbf{V}, \forall \boldsymbol{\mu} \in \mathbf{M}(s)
$$

where $\mathscr{L}(\cdot, \cdot)$ is the Lagrangian of the system defined as

$$
\mathscr{L}(\mathbf{v}, \boldsymbol{\mu})=\frac{1}{2} a(\mathbf{v}, \mathbf{v})-L(\mathbf{v})-b(\boldsymbol{\mu}, \mathbf{v}) .
$$

Another classical weak formulation of problem (1)-(6) is given by the following variational inequality [18]: find $\mathbf{u} \in \mathbf{K}$ such that

$$
a(\mathbf{u}, \mathbf{v}-\mathbf{u})+j(s, \mathbf{v})-j(s, \mathbf{u}) \geq L(\mathbf{v}-\mathbf{u}), \quad \forall \mathbf{v} \in \mathbf{K},
$$


where $j(s, \mathbf{v})=\left\langle s,\left|\llbracket v_{t} \rrbracket\right|\right\rangle_{W_{T}^{\prime}, W_{T}}$ and $\mathbf{K}$ denotes the closed convex cone of admissible displacement fields satisfying the non-interpenetration condition

$$
\mathbf{K}=\left\{\mathbf{v} \in \mathbf{V}: \quad \llbracket v_{n} \rrbracket \leq 0 \text { on } \Gamma_{C}\right\} .
$$

Moreover, the first argument $\mathbf{u}$ solution to (7) is also the unique solution to problem (9) and one has $\lambda_{n}=\sigma_{n}(\mathbf{u})$ in $W_{N}^{\prime}$ and $\lambda_{t}=\sigma_{t}(\mathbf{u})$ in $W_{T}^{\prime}$.

\section{Discretization with the stabilized Lagrange multiplier method}

\subsection{The discrete problem}

We shall now describe the enriched finite elements used in the approximation of the mixed problem (7). For any given discretization parameter $h>0$, let $\mathcal{T}^{h}$, be a partition of the uncracked domain $\bar{\Omega}$ with a maximal size $h, \bar{\Omega}=\bigcup_{T \in \mathcal{T}^{h}} \bar{T}$. Moreover, $\mathcal{T}^{h}$ is assumed to be regular, i.e., there exists $\beta>0$ such that $\forall T \in \mathcal{T}^{h}, h_{T} / \rho_{T} \leq \beta$ where $\rho_{T}$ denotes the radius of the inscribed circle in $T$ (see [15]). We consider the variant, called the cut-off XFEM, introduced in [14] in which the whole area around the crack tip is enriched by using a cut-off function denoted by $\chi(\cdot)$. In this variant, the enriched finite-element space $\mathbf{V}^{h}$ is defined as

$$
\mathbf{V}^{h}=\left\{\mathbf{v}^{h} \in(\mathscr{C}(\bar{\Omega}))^{2}: \mathbf{v}^{h}=\sum_{i \in \mathcal{N}_{h}} \mathbf{a}_{i} \varphi_{i}+\sum_{i \in \mathcal{N}_{h}^{H}} \mathbf{b}_{i} H \varphi_{i}+\chi \sum_{j=1}^{4} \mathbf{c}_{j} F_{j}, \quad \mathbf{a}_{i}, \mathbf{b}_{i}, \mathbf{c}_{j} \in \mathbb{R}^{2}\right\} \subset \mathbf{V}
$$

Here $(\mathscr{C}(\bar{\Omega}))^{2}$ is the space of continuous vector fields over $\bar{\Omega}, H(\cdot)$ is the Heaviside-like function used to represent the discontinuity across the straight crack and defined by

$$
H(\mathbf{x})= \begin{cases}+1 & \text { if }\left(\mathbf{x}-\mathbf{x}^{*}\right) \cdot \mathbf{n}^{+} \geq 0 \\ -1 & \text { otherwise }\end{cases}
$$

where $\mathbf{x}^{*}$ denotes the position of the crack tip. The notation $\varphi_{i}$ represents the scalar-valued shape function associated with the classical degree one finite-element method at the node of index $i, \mathcal{N}_{h}$ denotes the set of all node indices, and $\mathcal{N}_{h}^{H}$ denotes the set of node indices enriched by the function $H(\cdot)$, i.e., node indices for which the support of the corresponding shape function is completely cut by the crack (see Fig. 2). The cut-off function is a $\mathscr{C}^{1}$ piecewise third order polynomial on $\left[r_{0}, r_{1}\right]$ such that:

$$
\begin{cases}\chi(r)=1 & \text { if } r<r_{0} \\ \chi(r) \in(0,1) & \text { if } r_{0}<r<r_{1} \\ \chi(r)=0 & \text { if } r>r_{1}\end{cases}
$$

In our case we take $\chi(r)=\frac{2 r^{3}-3\left(r_{0}+r_{1}\right) r^{2}+6 r_{1} r_{0} r+\left(r_{0}-3 r_{1}\right) r_{0}^{2}}{\left(r_{0}-r_{1}\right)^{3}}$ if $r_{0} \leq r \leq r_{1}$ with $r_{0}=0.01$ and $r_{1}=0.49$. The functions $\left\{F_{j}(\mathbf{x})\right\}_{1 \leq j \leq 4}$ are defined in polar coordinates located at the crack tip by

$$
\left\{F_{j}(\mathbf{x}), 1 \leq j \leq 4\right\}=\left\{\sqrt{r} \sin \frac{\theta}{2}, \sqrt{r} \cos \frac{\theta}{2}, \sqrt{r} \sin \frac{\theta}{2} \sin \theta, \sqrt{r} \cos \frac{\theta}{2} \sin \theta\right\} .
$$

These functions allow to generate the asymptotic non-smooth displacement at the crack tip (see $[25])$. 


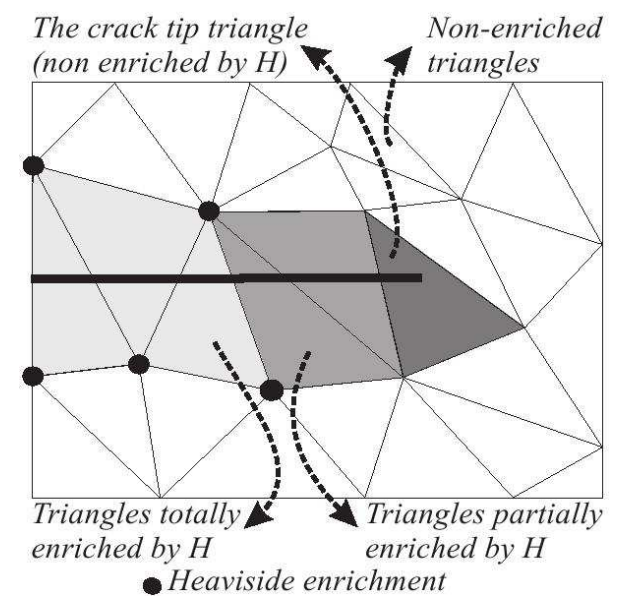

Figure 2: A cracked domain.

An important point of the approximation is whether the normal and tangent contact pressure $\left(\sigma_{n}\right.$ and $\left.\sigma_{t}\right)$ are regular or not at the crack lips. If it were singular, it should be taken into account by the discretization of the multiplier. If we assume that there is a finite number of transition points between contact and non contact zones and slip and non slip zones on the crack lips, we are able to prove (similarly to the case of frictionless contact presented in [3]) that the normal and tangent contact stress $\sigma_{n}$ and $\sigma_{t}$ are in $H^{1 / 2}\left(\Gamma_{C}\right)$ (see Lemma B.7 in Appendix B).

Now, concerning the discretization of the multiplier, let $\mathbf{x}_{0}, \ldots, \mathbf{x}_{N}$ be given distinct points lying in $\overline{\Gamma_{C}}$ and coming from the intersection between $\mathcal{T}^{h}$ and $\overline{\Gamma_{C}}$. These nodes form a one-dimensional family of meshes of $\Gamma_{C}$ denoted by $S^{h}$. The mesh $S^{h}$ allows us to define a finite-dimensional space $W^{h}$ approximating $\left(W_{N}\right)^{\prime}$ or $\left(W_{T}\right)^{\prime}$ and a nonempty closed convex set $M_{N}^{h} \subset W^{h}$ (resp. $\left.M_{T}^{h}(s) \subset W^{h}\right)$ approximating $M_{N}\left(\operatorname{resp} M_{T}(s)\right)$. We consider two possible elementary choices of $W^{h}$ :

$$
\begin{aligned}
& W_{0}^{h}=\left\{\mu^{h} \in L^{2}\left(\Gamma_{C}\right):\left.\mu_{\mid}^{h}\right|_{\left(\mathbf{x}_{i}, \mathbf{x}_{i+1}\right)} \in P_{0}\left(\mathbf{x}_{i}, \mathbf{x}_{i+1}\right), \forall 0 \leq i \leq N-1\right\}, \\
& W_{1}^{h}=\left\{\mu^{h} \in \mathscr{C}\left(\Gamma_{C}\right):\left.\mu^{h}\right|_{\left(\mathbf{x}_{i}, \mathbf{x}_{i+1}\right)} \in P_{1}\left(\mathbf{x}_{i}, \mathbf{x}_{i+1}\right), \forall 0 \leq i \leq N-1\right\},
\end{aligned}
$$

where $P_{k}(E)$ denotes the space of polynomials of degree less or equal to $k$ on $E$. This allows to provide the following three elementary definitions of $M_{N}^{h}$ and $M_{T}^{h}(s)$ :

$$
\begin{gathered}
M_{N 0}^{h}=\left\{\mu^{h} \in W_{0}^{h}: \mu^{h} \leq 0 \text { on } \Gamma_{C}\right\} \\
M_{T 0}^{h}(s)=\left\{\mu^{h} \in W_{0}^{h}:\left|\mu^{h}\right| \leq s \text { on } \Gamma_{C}\right\} \\
M_{N 1}^{h}=\left\{\mu^{h} \in W_{1}^{h}: \mu^{h} \leq 0 \text { on } \Gamma_{C}\right\}, \\
M_{T 1}^{h}(s)=\left\{\mu^{h} \in W_{1}^{h}:\left|\mu^{h}\right| \leq s \text { on } \Gamma_{C}\right\}, \\
M_{N 1, *}^{h}=\left\{\mu^{h} \in W_{1}^{h}: \int_{\Gamma_{C}} \mu^{h} \psi^{h} d \Gamma \geq 0, \forall \psi^{h} \in M_{N 1}^{h}\right\} .
\end{gathered}
$$




$$
M_{T 1, *}^{H}(s)=\left\{\mu^{h} \in W_{1}^{h}: \int_{\Gamma_{C}} \mu^{h} \psi^{h} d \Gamma+s \int_{\Gamma_{C}}\left|\psi^{h}\right| d \Gamma \geq 0, \forall \psi^{H} \in W_{1}^{h}\right\} .
$$

Let $\mathbf{W}^{h}=W^{h} \times W^{h}$. In the forthcoming convergence analysis, we will need more information on the compatibility between the spaces $\mathbf{V}^{h}$ and $\mathbf{W}^{h}$. To overcome this difficulty, we use the local projection stabilization technique introduced in [5]. This technique consists in adding a supplementary term, involving the local orthogonal $L^{2}$ projection of the multiplier on a patch decomposition of the mesh, to the discrete mixed formulation. The set of patches is build from $S^{h}$. Indeed we aggregate the possibly very small elements of $S^{h}$ in order to obtain a set of patches having a minimal and a maximal size (for instance between $3 h$ and $6 h$ ). In practice, this operation can be done rather easily (even for three-dimensional problems). A practical way to obtain such a patch decomposition will be described in the next section. An example of patch aggregation is presented in Fig. 3. Let $H$ be the maximum length of these patches and denote by $S^{H}$ the corresponding subdivision of $\Gamma_{C}$. Let

$$
W^{H}=\left\{\mu^{H} \in L^{2}\left(\Gamma_{C}\right): \mu^{H}{ }_{S} \in P_{0}(S), \forall S \in S^{H}\right\},
$$

be the space of piecewise constants on this mesh and let $\mathbf{W}^{H}=W^{H} \times W^{H}$. Similarly to the classical result presented in [16], we prove that an inf-sup condition is satisfied between $\mathbf{V}^{h}$ and $\mathbf{W}^{H}$ for minimal size of $3 h$ for the patches (see Appendix A for the proof on a scalar field, which can be straightforwardly generalized to vector field). This implies in particular that an optimal convergence can be reached if the multiplier is taken in $\mathbf{W}^{H}$. However, this suppose a relatively coarse approximation of the multiplier. Our approach is to use this result in order to stabilize the approximation obtained with the multiplier defined on the finer discretization $\mathbf{W}^{h}$. Let us first recall the result in Lemma A.1 Appendix A. Under a condition for the patches $S \in S^{H}$ to be approximated by a fixed set of line segments having approximatively the same length with a length greater or equal to $3 h$, the following inf-sup (or LBB) condition holds for a constant $\beta^{*}>0$, independent of $h$ and $H$ :

$$
\forall \boldsymbol{\mu}^{H} \in \mathbf{W}^{H}, \quad \sup _{\mathbf{v}^{h} \in \mathbf{V}^{h}} \frac{b\left(\boldsymbol{\mu}^{H}, \mathbf{v}^{h}\right)}{\left\|\mathbf{v}^{h}\right\|} \geq \beta^{*}\left\|\boldsymbol{\mu}^{H}\right\| \mathbf{W}^{\prime} .
$$

In the following, we will assume that the conditions guaranteeing this inf-sup condition are satisfied. 


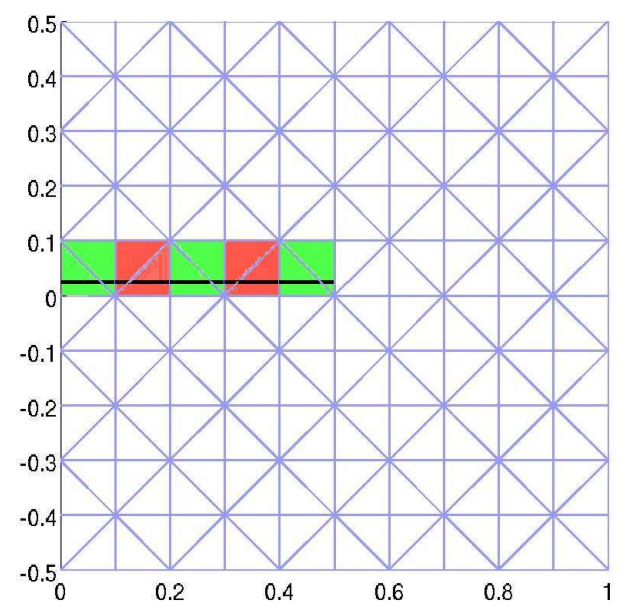

Figure 3: Example of a patch aggregation (in red and green) of size approximatively $h$ of the intersection between the crack and the mesh.

Let $P_{W^{H}}$ be the local orthogonal projection operator from $L^{2}\left(\Gamma_{C}\right)$ onto $W^{H}$ which is defined by

$$
\forall \mu \in L^{2}\left(\Gamma_{C}\right), \forall S \in S^{H} \quad P_{W^{H}}(\mu)_{\mid S}=\frac{1}{\operatorname{mes}(S)} \int_{S} \mu d \Gamma
$$

and $P_{\mathbf{W}^{H}}$ be the local vector orthogonal projection operator from $L^{2}\left(\Gamma_{C}\right) \times L^{2}\left(\Gamma_{C}\right)$ onto $\mathbf{W}^{H}$ which is defined by $P_{\mathbf{W}^{H}}(\boldsymbol{\mu})=\left(P_{W^{H}}\left(\mu_{n}\right), P_{W^{H}}\left(\mu_{t}\right)\right), \forall \boldsymbol{\mu}=\left(\mu_{n}, \mu_{t}\right) \in L^{2}\left(\Gamma_{C}\right) \times L^{2}\left(\Gamma_{C}\right)$. The stabilized formulation consists in replacing the Lagrangian (8) by the following one:

$\mathcal{L}_{h}\left(v^{h}, \mu^{h}\right)=\frac{1}{2} a\left(\mathbf{v}^{h}, \mathbf{v}^{h}\right)-L\left(\mathbf{v}^{h}\right)-b\left(\boldsymbol{\mu}^{h}, \mathbf{v}^{h}\right)-\frac{\gamma}{2} \int_{\Gamma_{c}}\left(\mu_{n}^{h}-P_{W^{H}}\left(\mu_{n}^{h}\right)\right)^{2} d \Gamma-\frac{\gamma}{2} \int_{\Gamma_{c}}\left(\mu_{t}^{h}-P_{W^{H}}\left(\mu_{t}^{h}\right)\right)^{2} d \Gamma$,

where, $\gamma$ is a constant independent of the mesh size. Let $\mathbf{M}^{h}(s)=M_{N}^{h} \times M_{T}^{h}(s)$ then the corresponding optimality system reads as follows:

(18)

$\left\{\begin{array}{l}\text { Find } u^{h} \in V^{h} \text { and } \boldsymbol{\lambda}^{h}=\left(\lambda_{n}^{h}, \lambda_{t}^{h}\right) \in M^{h}(s) \text { such that: } \\ a\left(\mathbf{u}^{h}, \mathbf{v}^{h}\right)-b\left(\lambda^{h}, \mathbf{v}^{h}\right)=L\left(\mathbf{v}^{h}\right), \quad \forall \mathbf{v}^{h} \in \mathbf{V}^{h}, \\ b\left(\boldsymbol{\mu}^{h}-\boldsymbol{\lambda}^{h}, \mathbf{u}^{h}\right)+\gamma \int_{\Gamma_{c}}\left(\lambda_{n}^{h}-P_{W^{H}}\left(\lambda_{n}^{h}\right)\right)\left(\left(\mu_{n}^{h}-\lambda_{n}^{h}\right)-\left(P_{W^{H}}\left(\mu_{n}^{h}\right)-P_{W^{H}}\left(\lambda_{n}^{h}\right)\right)\right) d \Gamma \\ \quad+\gamma \int_{\Gamma_{c}}\left(\lambda_{t}^{h}-P_{W^{H}}\left(\lambda_{t}^{h}\right)\right)\left(\left(\mu_{t}^{h}-\lambda_{t}^{h}\right)-\left(P_{W^{H}}\left(\mu_{t}^{h}\right)-P_{W^{H}}\left(\lambda_{t}^{h}\right)\right)\right) d \Gamma \geq 0, \forall \boldsymbol{\mu}^{h}=\left(\mu_{n}^{h}, \mu_{t}^{h}\right) \in \mathbf{M}^{h}(s) .\end{array}\right.$

\subsection{Existence and uniqueness of the solution to the stabilized problem}

Lemma 3.1. Assume that (17) holds then for any $\gamma>0$ there exists a unique solution of the stabilized problem (18).

Proof. Let $\boldsymbol{\mu}^{h}=\left(\mu_{n}^{h}, \mu_{t}^{h}\right) \in \mathbf{M}^{h}(s)$ and $\mathbf{u}^{h}$ be the solution of the following problem:

$$
a\left(\mathbf{u}^{h}, \mathbf{v}^{h}\right)-L\left(\mathbf{v}^{h}\right)=b\left(\boldsymbol{\mu}^{h}, \mathbf{v}^{h}\right)=\left\langle\mu_{n}^{h}, \llbracket v_{n}^{h} \rrbracket\right\rangle_{W^{h^{\prime}}, W^{h}}+\left\langle\mu_{t}^{h}, \llbracket v_{t}^{h} \rrbracket\right\rangle_{W^{h^{\prime}}, W^{h}} \quad \forall \mathbf{v}^{h} \in \mathbf{V}^{h},
$$


then using the fact that the inf-sup condition is satisfied in the orthogonal of the kernel of $b(.,$. (which contains $\mathbf{W}^{h}$ ) we prove that there exists a constant $C$ such that:

$$
C\left\|\mathbf{u}^{h}\right\|+\|L\|_{V^{\prime}} \geq\left\|P_{\mathbf{W}^{H}}\left(\boldsymbol{\mu}^{h}\right)\right\|_{\mathbf{W}^{\prime}} .
$$

We have

$$
\begin{aligned}
\mathcal{L}_{h}\left(\mathbf{u}^{h}, \boldsymbol{\mu}^{h}\right)= & \frac{1}{2} a\left(\mathbf{u}^{h}, \mathbf{u}^{h}\right)-L\left(\mathbf{u}^{h}\right)-b\left(\boldsymbol{\mu}^{h}, \mathbf{u}^{h}\right)-\frac{\gamma}{2} \int_{\Gamma_{C}}\left(\mu_{n}^{h}-P_{W^{H}}\left(\mu_{n}^{h}\right)\right)^{2} d \Gamma \\
& -\frac{\gamma}{2} \int_{\Gamma_{C}}\left(\mu_{t}^{h}-P_{W^{H}}\left(\mu_{t}^{h}\right)\right)^{2} d \Gamma, \\
= & -\frac{1}{2} a\left(\mathbf{u}^{h}, \mathbf{u}^{h}\right)-\frac{\gamma}{2} \int_{\Gamma_{C}}\left(\mu_{n}^{h}-P_{W^{H}}\left(\mu_{n}^{h}\right)\right)^{2} d \Gamma-\frac{\gamma}{2} \int_{\Gamma_{C}}\left(\mu_{t}^{h}-P_{W^{H}}\left(\mu_{t}^{h}\right)\right)^{2} d \Gamma, \\
= & -\frac{1}{2}\left\|\mathbf{u}^{h}\right\|^{2}-\frac{\gamma}{2}\left\|\mu_{n}^{h}-P_{W^{H}}\left(\mu_{n}^{h}\right)\right\|_{0, \Gamma_{C}}^{2}-\frac{\gamma}{2}\left\|\mu_{t}^{h}-P_{W^{H}}\left(\mu_{t}^{h}\right)\right\|_{0, \Gamma_{C}}^{2} .
\end{aligned}
$$

When $\left\|\boldsymbol{\mu}^{h}\right\|_{\mathbf{W}^{\prime}} \rightarrow \infty$ we have $\left\|P_{\mathbf{W}^{H}}\left(\boldsymbol{\mu}^{h}\right)\right\|_{\mathbf{W}^{\prime}} \rightarrow \infty$ (using inequality (19) we have $\left\|\mathbf{u}^{h}\right\| \rightarrow \infty$ ) or/and $\left\|\boldsymbol{\mu}^{h}-P_{\mathbf{W}^{H}}\left(\boldsymbol{\mu}^{h}\right)\right\|_{\mathbf{W}^{\prime}} \rightarrow \infty$, which implies that

$$
\lim _{\boldsymbol{\mu}^{h} \in \mathbf{M}^{h}(s),\left\|\boldsymbol{\mu}^{h}\right\|_{\mathbf{W}^{\prime}} \rightarrow \infty} \mathcal{L}_{\gamma}\left(\mathbf{u}^{h}, \boldsymbol{\mu}^{h}\right)=-\infty .
$$

Now the existence of a solution to Problem (18) follows from the fact that $\mathbf{V}^{h}$ and $\mathbf{M}^{h}(s)$ are two nonempty closed convex sets, $\mathcal{L}_{\gamma}(\cdot, \cdot)$ is continuous on $\mathbf{V}^{h} \times \mathbf{W}^{h}, \mathcal{L}_{\gamma}\left(\mathbf{v}^{h}\right.$, .) (resp. $\mathcal{L}_{\gamma}\left(\cdot, \boldsymbol{\mu}^{h}\right)$ ) is concave (resp. strictly convex) for any $\mathbf{v}^{h} \in \mathbf{V}^{h}$ (resp. for any $\mu^{h} \in \mathbf{M}^{h}$ ) and $\lim _{\mathbf{v}^{h} \in \mathbf{V}^{h},\left\|\mathbf{v}^{h}\right\| \rightarrow \infty} \mathcal{L}_{\gamma}\left(\mathbf{v}^{h}, 0\right)=+\infty\left(\right.$ resp. $\left.\lim _{\boldsymbol{\mu}^{h} \in \mathbf{M}^{h}(s),\left\|\boldsymbol{\mu}^{h}\right\|_{\mathbf{w}^{\prime} \rightarrow \infty}} \mathcal{L}_{\gamma}\left(\mathbf{u}^{h}, \boldsymbol{\mu}^{h}\right)=-\infty\right)$, see [19, pp. 338-339]. The strict convexity of $a(.,$.$) implies the uniqueness of the first argument \mathbf{u}^{h}$. Now let $\boldsymbol{\lambda}_{1}^{h}$ and $\boldsymbol{\lambda}_{2}^{h}$ two solutions of (18) then we have:

$$
\begin{aligned}
b\left(\boldsymbol{\lambda}_{2}^{h}-\boldsymbol{\lambda}_{1}^{h}, \mathbf{u}^{h}\right)+ & \gamma \int_{\Gamma_{c}}\left(\lambda_{1 n}^{h}-P_{W^{H}}\left(\lambda_{1 n}^{h}\right)\right)\left(\left(\lambda_{2 n}^{h}-\lambda_{1 n}^{h}\right)-\left(P_{W^{H}}\left(\lambda_{1 n}^{h}\right)-P_{W^{H}}\left(\lambda_{2 n}^{h}\right)\right)\right) d \Gamma \\
& +\gamma \int_{\Gamma_{c}}\left(\lambda_{1 t}^{h}-P_{W^{H}}\left(\lambda_{1 t}^{h}\right)\right)\left(\left(\lambda_{2 t}^{h}-\lambda_{1 t}^{h}\right)-\left(P_{W^{H}}\left(\lambda_{1 t}^{h}\right)-P_{W^{H}}\left(\lambda_{2 t}^{h}\right)\right)\right) d \Gamma \geq 0, \\
b\left(\boldsymbol{\lambda}_{1}^{h}-\lambda_{2}^{h}, \mathbf{u}^{h}\right)+ & \gamma \int_{\Gamma_{c}}\left(\lambda_{2 n}^{h}-P_{W^{H}}\left(\lambda_{2 n}^{h}\right)\right)\left(\left(\lambda_{1 n}^{h}-\lambda_{2 n}^{h}\right)-\left(P_{W^{H}}\left(\lambda_{2 n}^{h}\right)-P_{W^{H}}\left(\lambda_{1 n}^{h}\right)\right)\right) \\
& +\gamma \int_{\Gamma_{c}}\left(\lambda_{2 t}^{h}-P_{W^{H}}\left(\lambda_{2 t}^{h}\right)\right)\left(\left(\lambda_{1 t}^{h}-\lambda_{2 t}^{h}\right)-\left(P_{W^{H}}\left(\lambda_{2 t}^{h}\right)-P_{W^{H}}\left(\lambda_{1 t}^{h}\right)\right)\right) d \Gamma \geq 0,
\end{aligned}
$$

and by summation of the last two inequalities we have:

$\left\|\left(\lambda_{1 n}^{h}-\lambda_{2 n}^{h}\right)-\left(P_{W^{H}}\left(\lambda_{1 n}^{h}\right)-P_{W^{H}}\left(\lambda_{2 n}^{h}\right)\right)\right\|_{0, \Gamma_{C}}^{2}+\left\|\left(\lambda_{1 t}^{h}-\lambda_{2 t}^{h}\right)-\left(P_{W^{H}}\left(\lambda_{1 t}^{h}\right)-P_{W^{H}}\left(\lambda_{2 t}^{h}\right)\right)\right\|_{0, \Gamma_{C}}^{2} \leq 0$, therefore $\lambda_{1 n}^{h}-\lambda_{2 n}^{h}=P_{W^{H}}\left(\lambda_{2 n}^{h}\right)-P_{W^{H}}\left(\lambda_{1 n}^{h}\right)$ and $\lambda_{1 t}^{h}-\lambda_{2 t}^{h}=P_{W^{H}}\left(\lambda_{2 t}^{h}\right)-P_{W^{H}}\left(\lambda_{1 t}^{h}\right)$ (i.e. $\lambda_{1 n}^{h}-\lambda_{2 n}^{h} \in W^{H}$ and $\left.\lambda_{1 t}^{h}-\lambda_{2 t}^{h} \in W^{H}\right)$. Let $\overline{\boldsymbol{\lambda}}^{h}=\left(\lambda_{1 n}^{h}-\lambda_{2 n}^{h}, \lambda_{1 t}^{h}-\lambda_{2 t}^{h}\right)$ and $\overline{\boldsymbol{\lambda}}^{H}=\left(P_{W^{H}}\left(\lambda_{1 n}^{h}\right)-\right.$ $\left.P_{W^{H}}\left(\lambda_{2 n}^{h}\right), P_{W^{H}}\left(\lambda_{1 t}^{h}\right)-P_{W^{H}}\left(\lambda_{2 t}^{h}\right)\right)$. From inequality (17) there exists $\mathbf{v}^{h} \in \mathbf{V}^{h}$ such that

$$
b\left(\overline{\boldsymbol{\lambda}}^{H}, \mathbf{v}^{h}\right) \geq \beta^{*}\left\|\overline{\boldsymbol{\lambda}}^{H}\right\| \mathbf{w}^{\prime}\left\|\mathbf{v}^{h}\right\|,
$$

and thus

$$
\beta^{*}\left\|\overline{\boldsymbol{\lambda}}^{H}\right\|_{\mathbf{W}^{\prime}} \leq \frac{1}{\left\|\mathbf{v}^{h}\right\|} b\left(\overline{\boldsymbol{\lambda}}^{H}, \mathbf{v}^{h}\right)=\frac{1}{\left\|\mathbf{v}^{h}\right\|} b\left(\overline{\boldsymbol{\lambda}}^{h}, \mathbf{v}^{h}\right)=\frac{1}{\left\|\mathbf{v}^{h}\right\|} a\left(\overline{\mathbf{u}}^{h}, \mathbf{v}^{h}\right)=0 .
$$

This implies the uniqueness of the second argument $\boldsymbol{\lambda}^{h}$, therefore (18) has a unique solution. 


\subsection{Convergence analysis}

In order to perform the error analysis, we recall the definition of the XFEM interpolation operator $\Pi^{h}$ introduced in [26].

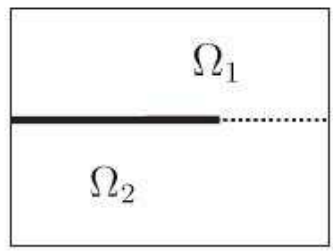

Figure 4: Decomposition of $\Omega$ into $\Omega_{1}$ and $\Omega_{2}$.

We assume that the displacement has the regularity $\left(H^{2}(\Omega)\right)^{2}$ except in the vicinity of the crack-tip where the singular part of the displacement is a linear combination of the functions $\left\{F_{j}(\mathbf{x})\right\}_{1 \leq j \leq 4}$ given by (10) (see [17] for a justification). Let us denote by $\mathbf{u}_{s}$ the singular part of $\mathbf{u}, \mathbf{u}_{r}=\mathbf{u}-\chi \mathbf{u}_{s}$ the regular part of $\mathbf{u}$, and $\mathbf{u}_{r}^{k}$ the restriction of $\mathbf{u}_{r}$ to $\Omega_{k}, k \in\{1,2\}$. Then, for $k \in\{1,2\}$, there exists an extension $\widetilde{\mathbf{u}}_{r}^{k} \in\left(H^{2}(\bar{\Omega})\right)^{2}$ of $\mathbf{u}_{r}^{k}$ to $\bar{\Omega}$ such that (see [1])

$$
\begin{aligned}
\left\|\widetilde{\mathbf{u}}_{r}^{1}\right\|_{2, \bar{\Omega}} & \lesssim\left\|\mathbf{u}_{r}^{1}\right\|_{2, \Omega_{1}}, \\
\left\|\widetilde{\mathbf{u}}_{r}^{2}\right\|_{2, \bar{\Omega}} & \lesssim\left\|\mathbf{u}_{r}^{2}\right\|_{2, \Omega_{2}} .
\end{aligned}
$$

Here and throughout the paper, we use the notation $a \lesssim b$ to signify that there exists a constant $C>0$, independent of the mesh parameter $h$, the solution and the position of the crack-tip, such that $a \leq C b$.

Definition $3.2([26])$. Given a displacement field $\mathbf{u}$ satisfying $\mathbf{u}-\mathbf{u}_{s} \in H^{2}(\Omega)$, and two extensions $\widetilde{\mathbf{u}}_{r}^{1}$ and $\widetilde{\mathbf{u}}_{r}^{2}$ in $H^{2}(\bar{\Omega})$ of $\mathbf{u}_{r}^{1}$ and $\mathbf{u}_{r}^{2}$, respectively, we define $\Pi^{h} \mathbf{u}$ as the element of $\mathbf{V}^{h}$ such that

$$
\Pi^{h} \mathbf{u}=\sum_{i \in \mathcal{N}_{h}} \mathbf{a}_{i} \varphi_{i}+\sum_{i \in \mathcal{N}_{h}^{H}} \mathbf{b}_{i} H \varphi_{i}+\chi \mathbf{u}_{s}
$$

where $\mathbf{a}_{i}, \mathbf{b}_{i}$ are given as follows for $\mathbf{y}_{i}$ the finite-element node associated to $\varphi_{i}$ :

$$
\begin{aligned}
& \text { if } i \in\left\{\mathcal{N}_{h} \backslash \mathcal{N}_{h}^{H}\right\} \text { then } \mathbf{a}_{i}=\mathbf{u}_{r}\left(\mathbf{y}_{i}\right), \\
& \text { if } i \in \mathcal{N}_{h}^{H} \text { and } \mathbf{y}_{i} \in \bar{\Omega}_{k} \text { for } k \in\{1,2\} \text { then for } l=3-k: \\
& \left\{\begin{array}{l}
\mathbf{a}_{i}=\frac{1}{2}\left(\mathbf{u}_{r}^{k}\left(\mathbf{y}_{i}\right)+\widetilde{\mathbf{u}}_{r}^{l}\left(\mathbf{y}_{i}\right)\right), \\
\mathbf{b}_{i}=\frac{(-1)^{k}}{2}\left(\mathbf{u}_{r}^{k}\left(\mathbf{y}_{i}\right)-\widetilde{\mathbf{u}}_{r}^{l}\left(\mathbf{y}_{i}\right)\right) .
\end{array}\right.
\end{aligned}
$$

This XFEM interpolation operator satisfies the following interpolation error estimate [26]:

$$
\left\|\mathbf{u}-\Pi^{h} \mathbf{u}\right\| \lesssim h\left\|\mathbf{u}-\chi \mathbf{u}_{s}\right\|_{2, \Omega} .
$$


Lemma 3.3. Let $(\mathbf{u}, \boldsymbol{\lambda}) \in V \times \mathbf{M}(s)$ be the solution of (7) and $\left(\mathbf{u}^{h}, \boldsymbol{\lambda}^{h}\right) \in \mathbf{V}^{h} \times \mathbf{M}^{h}(s)$ be the solution of (18). Then we have:

$$
\begin{aligned}
\alpha\left\|\mathbf{u}^{h}-\mathbf{u}\right\|^{2} \leq & M\left\|\mathbf{u}^{h}-\mathbf{u}\right\|\left\|\mathbf{v}^{h}-\mathbf{u}\right\|+\left\|\lambda_{n}^{h}-\lambda_{n}\right\| \mathbf{W}^{\prime}\left\|\mathbf{u}-\mathbf{v}^{h}\right\|+\left\|\lambda_{t}^{h}-\lambda_{t}\right\| \mathbf{W}^{\prime}\left\|\mathbf{u}-\mathbf{v}^{h}\right\| \\
& +b\left(\boldsymbol{\mu}^{h}-\boldsymbol{\lambda}, \mathbf{u}^{h}-\mathbf{u}\right)+b\left(\boldsymbol{\lambda}^{h}-\boldsymbol{\mu}^{h}, \mathbf{u}^{h}\right)+b\left(\boldsymbol{\mu}^{h}-\boldsymbol{\lambda}, \mathbf{u}\right) \\
& +b\left(\boldsymbol{\lambda}-\boldsymbol{\lambda}^{h}, \mathbf{u}\right), \quad \forall \mathbf{v}^{h} \in \mathbf{V}^{h}, \boldsymbol{\mu}^{h} \in \mathbf{W}^{h}, \\
(22) \quad & 8 M^{2}\left\|\mathbf{u}-\mathbf{u}^{h}\right\|^{2}+8 \beta^{* 2}\left\|\boldsymbol{\lambda}-\overline{\boldsymbol{\mu}}^{H}\right\|_{\mathbf{W}^{\prime}}^{2}+8\left\|\boldsymbol{\lambda}-\overline{\boldsymbol{\mu}}^{h}\right\|_{\mathbf{W}^{\prime}}^{2} \\
\beta^{* 2}\left\|\boldsymbol{\lambda}^{H}-\boldsymbol{\lambda}\right\|_{\mathbf{W}^{\prime}}^{2} \leq & +8\left\|\overline{\boldsymbol{\mu}}^{H}-\overline{\boldsymbol{\mu}}^{h}\right\|_{\mathbf{W}^{\prime}}^{2}+8\left\|\boldsymbol{\lambda}^{H}-\boldsymbol{\lambda}^{h}\right\|_{\mathbf{W}^{\prime}}^{2} \quad \forall \overline{\boldsymbol{\mu}}^{h}=\left(\bar{\mu}_{n}^{h}, \bar{\mu}_{t}^{h}\right) \in \mathbf{W}^{h},
\end{aligned}
$$

with $\boldsymbol{\lambda}^{H}=P_{\mathbf{W}^{H}}\left(\boldsymbol{\lambda}^{h}\right), \overline{\boldsymbol{\mu}}^{H}=P_{\mathbf{W}^{H}}\left(\overline{\boldsymbol{\mu}}^{h}\right)$.

Proof. For all $\mathbf{v}^{h} \in \mathbf{V}^{h}, \boldsymbol{\mu}^{h} \in \mathbf{W}^{h}$ one has

$$
\begin{aligned}
a\left(\mathbf{u}^{h}-\mathbf{u}, \mathbf{u}^{h}-\mathbf{u}\right)= & a\left(\mathbf{u}^{h}-\mathbf{u}, \mathbf{v}^{h}-\mathbf{u}\right)+a\left(\mathbf{u}^{h}-\mathbf{u}, \mathbf{u}^{h}-\mathbf{v}^{h}\right), \\
= & a\left(\mathbf{u}^{h}-\mathbf{u}, \mathbf{v}^{h}-\mathbf{u}\right)+b\left(\boldsymbol{\lambda}^{h}-\boldsymbol{\lambda}, \mathbf{u}^{h}-\mathbf{v}^{h}\right), \\
= & a\left(\mathbf{u}^{h}-\mathbf{u}, \mathbf{v}^{h}-\mathbf{u}\right)+b\left(\boldsymbol{\lambda}^{h}-\boldsymbol{\lambda}, \mathbf{u}-\mathbf{v}^{h}\right)+b\left(\boldsymbol{\lambda}^{h}-\boldsymbol{\lambda}, \mathbf{u}^{h}-\mathbf{u}\right), \\
= & a\left(\mathbf{u}^{h}-\mathbf{u}, \mathbf{v}^{h}-\mathbf{u}\right)+b\left(\boldsymbol{\lambda}^{h}-\boldsymbol{\lambda}, \mathbf{u}-\mathbf{v}^{h}\right)+b\left(\boldsymbol{\mu}^{h}-\boldsymbol{\lambda}, \mathbf{u}^{h}-\mathbf{u}\right) \\
& +b\left(\boldsymbol{\lambda}^{h}-\boldsymbol{\mu}^{h}, \mathbf{u}^{h}\right)+b\left(\boldsymbol{\mu}^{h}-\boldsymbol{\lambda}, \mathbf{u}\right)+b\left(\boldsymbol{\lambda}-\boldsymbol{\lambda}^{h}, \mathbf{u}\right) .
\end{aligned}
$$

From the $\mathbf{V}$-ellipticity and the continuity of the bilinear form $a(.,$.$) we prove the first inequality$ of Lemma 3.3. Now we shall give an estimate of the second inequality of Lemma 3.3. By noticing that

$$
\int_{\Gamma_{C}}\left(\boldsymbol{\lambda}-\boldsymbol{\lambda}^{h}\right) \cdot \llbracket \mathbf{v}^{h} \rrbracket d \Gamma=a\left(\mathbf{u}^{h}-\mathbf{u}, \mathbf{v}^{h}\right) \quad \forall \mathbf{v}^{h} \in \mathbf{V}^{h}
$$

one obtains

$$
\begin{aligned}
\int_{\Gamma_{C}}\left(\overline{\boldsymbol{\mu}}^{h}-\boldsymbol{\lambda}^{h}\right) \cdot \llbracket \mathbf{v}^{h} \rrbracket d \Gamma= & a\left(\mathbf{u}^{h}-\mathbf{u}, \mathbf{v}^{h}\right) \\
& +\int_{\Gamma_{C}}\left(\overline{\boldsymbol{\mu}}^{h}-\boldsymbol{\lambda}\right) \cdot \llbracket \mathbf{v}^{h} \rrbracket d \Gamma, \forall\left(\mathbf{v}^{h}, \overline{\boldsymbol{\mu}}^{h}\right) \in \mathbf{V}^{h} \times \mathbf{W}^{h} .
\end{aligned}
$$

Now, for $\boldsymbol{\mu}^{H}=\boldsymbol{\lambda}^{H}-\overline{\boldsymbol{\mu}}^{H} \in \mathbf{W}^{H}$ with $\overline{\boldsymbol{\mu}}^{H} \in \mathbf{W}^{H}$ the inf-sup condition (17) ensures the existence of $\mathbf{v}^{h} \in \mathbf{V}^{h}$ such that together with (24) we get

$$
\begin{aligned}
\beta^{*}\left\|\boldsymbol{\lambda}^{H}-\overline{\boldsymbol{\mu}}^{H}\right\|_{\mathbf{w}^{\prime}} \leq & \frac{1}{\left\|\mathbf{v}^{h}\right\|} \int_{\Gamma_{C}}\left(\overline{\boldsymbol{\mu}}^{H}-\boldsymbol{\lambda}^{H}\right) \cdot \llbracket \mathbf{v}^{h} \rrbracket d \Gamma \\
= & \frac{1}{\left\|\mathbf{v}^{h}\right\|} \int_{\Gamma_{C}}\left(\overline{\boldsymbol{\mu}}^{h}-\boldsymbol{\lambda}^{h}\right) \cdot \llbracket \mathbf{v}^{h} \rrbracket d \Gamma+\frac{1}{\left\|\mathbf{v}^{h}\right\|} \int_{\Gamma_{C}}\left(\overline{\boldsymbol{\mu}}^{H}-\boldsymbol{\lambda}^{H}-\left(\overline{\boldsymbol{\mu}}^{h}-\boldsymbol{\lambda}^{h}\right)\right) \cdot \llbracket \mathbf{v}^{h} \rrbracket d \Gamma, \\
\leq & M\left\|\mathbf{u}^{h}-\mathbf{u}\right\|+\left\|\bar{\mu}_{n}^{h}-\lambda_{n}\right\|_{W_{N}^{\prime}}+\left\|\bar{\mu}_{n}^{H}-\lambda_{n}^{H}-\left(\bar{\mu}_{n}^{h}-\lambda_{n}^{h}\right)\right\|_{W_{N}^{\prime}} \\
& +\left\|\bar{\mu}_{t}^{h}-\lambda_{t}\right\|_{W_{T}^{\prime}}+\left\|\bar{\mu}_{t}^{H}-\lambda_{t}^{H}-\left(\bar{\mu}_{t}^{h}-\lambda_{t}^{h}\right)\right\|_{W_{T}^{\prime}} .
\end{aligned}
$$

As a consequence, one has

$$
\begin{aligned}
\beta^{*}\left\|\boldsymbol{\lambda}^{H}-\boldsymbol{\lambda}\right\|_{\mathbf{W}^{\prime}} \leq & \beta^{*}\left\|\boldsymbol{\lambda}-\overline{\boldsymbol{\mu}}^{H}\right\|_{\mathbf{W}^{\prime}}+M\left\|\mathbf{u}^{h}-\mathbf{u}\right\|+\left\|\bar{\mu}_{n}^{h}-\lambda_{n}\right\|_{W_{N}^{\prime}}+\left\|\bar{\mu}_{t}^{h}-\lambda_{t}\right\|_{W_{T}^{\prime}} \\
& +\left\|\bar{\mu}_{n}^{H}-\bar{\mu}_{n}^{h}\right\|_{W_{N}^{\prime}}+\left\|\lambda_{n}^{H}-\lambda_{n}^{h}\right\|_{W_{N}^{\prime}}+\left\|\bar{\mu}_{t}^{H}-\bar{\mu}_{t}^{h}\right\|_{W_{T}^{\prime}}+\left\|\lambda_{t}^{H}-\lambda_{t}^{h}\right\|_{W_{T}^{\prime}},
\end{aligned}
$$

and

$$
\begin{aligned}
\beta^{* 2}\left\|\boldsymbol{\lambda}^{H}-\boldsymbol{\lambda}\right\|_{\mathbf{W}^{\prime}}^{2} \leq & 8 M^{2}\left\|\mathbf{u}-\mathbf{u}^{h}\right\|^{2}+8 \beta^{* 2}\left\|\boldsymbol{\lambda}-\overline{\boldsymbol{\mu}}^{H}\right\|_{\mathbf{W}^{\prime}}^{2}+8\left\|\boldsymbol{\lambda}-\overline{\boldsymbol{\mu}}^{h}\right\|_{\mathbf{W}^{\prime}}^{2} \\
& +8\left\|\overline{\boldsymbol{\mu}}^{H}-\overline{\boldsymbol{\mu}}^{h}\right\|_{\mathbf{W}^{\prime}}^{2}+8\left\|\boldsymbol{\lambda}^{H}-\boldsymbol{\lambda}^{h}\right\|_{\mathbf{W}^{\prime}}^{2} \quad \forall \overline{\boldsymbol{\mu}}^{h} \in \mathbf{W}^{h}, \forall \overline{\boldsymbol{\mu}}^{H} \in \mathbf{W}^{H} .
\end{aligned}
$$


In order to estimate these terms, we need to distinguish the different contact conditions (i.e., we must specify the definition of $\mathbf{M}^{h}(s)$ ). We consider hereafter three different standard discrete contact conditions.

\subsubsection{Conforming piecewise discontinuous discretization for multiplier $M_{N}^{h}=M_{N 0}^{h}$ and $M_{T}^{h}(s)=M_{T 0}^{h}(s)$}

We first consider the case of non-positive discontinuous piecewise constant multipliers where $M_{N}^{h}$ is defined by (11) and $M_{T}^{h}(s)$ is defined by (12). It is a conforming discretization on multiplier as $M_{N 0}^{h} \subset M_{N}$ and $M_{T 0}^{h}(s) \subset M_{T}(s)$.

Theorem 3.4. Let $(\mathbf{u}, \boldsymbol{\lambda})$ be the solution to Problem (7). Assume that $\mathbf{u}_{r} \in\left(H^{2}(\Omega)\right)^{2}$ and $\boldsymbol{\lambda} \in\left(H^{1 / 2}\left(\Gamma_{C}\right)\right)^{2}$. Let $\left(\mathbf{u}^{h}, \boldsymbol{\lambda}^{H}\right)$ be the solution to the discrete problem (18) where $M_{N}^{h}=M_{N 0}^{h}$ and $M_{T}^{h}(s)=M_{T 0}^{h}(s)$. Then, for any $\eta>0$ we have

$$
\left\|\mathbf{u}-\mathbf{u}^{h}\right\|+\left\|\boldsymbol{\lambda}-\boldsymbol{\lambda}^{h}\right\|_{\mathbf{W}^{\prime}} \lesssim h\left\|\mathbf{u}-\chi \mathbf{u}_{s}\right\|_{2, \Omega}+H^{\frac{3}{4}-\frac{\eta}{2}}\left(\|\mathbf{u}\|_{3 / 2-\eta, \Omega}+\|\boldsymbol{\lambda}\|_{1 / 2, \Gamma_{C}}\right) .
$$

Proof. In (22) we choose $\boldsymbol{\mu}^{h}=P_{\mathbf{W}^{H}}(\boldsymbol{\lambda})=\left(P_{W^{H}}\left(\lambda_{n}\right), P_{W^{H}}\left(\lambda_{t}\right)\right)$. We recall that the operator $P_{W^{H}}$ is defined for any $v \in L^{2}\left(\Gamma_{C}\right)$ by

$$
P_{W^{H}}(v) \in W^{H}, \quad \int_{\Gamma_{C}}\left(v-P_{W^{H}}(v)\right) \mu^{H} d \Gamma=0, \quad \forall \mu^{H} \in W^{H},
$$

and satisfies the following error estimates for any $0 \leq r \leq 1$ (see [10])

$$
H^{-1 / 2}\left\|v-P_{W^{H}}(v)\right\|_{-1 / 2, \Gamma_{C}}+\left\|v-P_{W^{H}}(v)\right\|_{0, \Gamma_{C}} \lesssim H^{r}\|v\|_{r, \Gamma_{C}} .
$$

Clearly, $\boldsymbol{\mu}^{h} \in M^{h}(s)$ and using the inequality coming from (18) we have

$$
b\left(\boldsymbol{\lambda}^{h}-\boldsymbol{\mu}^{h}, \mathbf{u}^{h}\right) \leq-\gamma\left\|\boldsymbol{\lambda}^{h}-\boldsymbol{\lambda}^{H}\right\|_{0, \Gamma_{C}}^{2},
$$

with $\boldsymbol{\lambda}^{H}=P_{\mathbf{W}^{H}}\left(\boldsymbol{\lambda}^{h}\right)=\left(P_{W^{H}}\left(\lambda_{n}^{h}\right), P_{W^{H}}\left(\lambda_{t}^{h}\right)\right)$. Moreover

$$
\begin{aligned}
b\left(\boldsymbol{\mu}^{h}-\boldsymbol{\lambda}, \mathbf{u}^{h}-\mathbf{u}\right)=b\left(P_{\mathbf{W}^{H}}(\boldsymbol{\lambda})-\boldsymbol{\lambda}, \mathbf{u}^{h}-\mathbf{u}\right) \leq & \left\|P_{W^{H}}\left(\lambda_{n}\right)-\lambda_{n}\right\|_{W_{N}^{\prime}}\left\|\mathbf{u}^{h}-\mathbf{u}\right\| \\
& +\left\|P_{W^{H}}\left(\lambda_{t}\right)-\lambda_{t}\right\|_{W_{T}^{\prime}}\left\|\mathbf{u}^{h}-\mathbf{u}\right\|,
\end{aligned}
$$

and

$$
\begin{aligned}
b\left(\boldsymbol{\mu}^{h}-\boldsymbol{\lambda}, \mathbf{u}\right)= & \int_{\Gamma_{C}}\left(P_{W^{H}}\left(\lambda_{n}\right)-\lambda_{n}\right) \llbracket u_{n} \rrbracket d \Gamma+\int_{\Gamma_{C}}\left(P_{W^{H}}\left(\lambda_{t}\right)-\lambda_{t}\right) \llbracket u_{t} \rrbracket d \Gamma, \\
= & \int_{\Gamma_{C}}\left(P_{W^{H}}\left(\lambda_{n}\right)-\lambda_{n}\right)\left(\llbracket u_{n} \rrbracket-P_{W^{H}}\left(\llbracket u_{n} \rrbracket\right)\right) d \Gamma \\
& +\int_{\Gamma_{C}}\left(P_{W^{H}}\left(\lambda_{t}\right)-\lambda_{t}\right)\left(\llbracket u_{t} \rrbracket-P_{W^{H}}\left(\llbracket u_{t} \rrbracket\right)\right) d \Gamma, \\
\leq & \left\|P_{W^{H}}\left(\lambda_{n}\right)-\lambda_{n}\right\|_{0, \Gamma_{C}}\left\|\llbracket u_{n} \rrbracket-P_{W^{H}}\left(\llbracket u_{n} \rrbracket\right)\right\|_{0, \Gamma_{C}} \\
& +\left\|P_{W^{H}}\left(\lambda_{t}\right)-\lambda_{t}\right\|_{0, \Gamma_{C}}\left\|\llbracket u_{t} \rrbracket-P_{W^{H}}\left(\llbracket u_{t} \rrbracket\right)\right\|_{0, \Gamma_{C}} .
\end{aligned}
$$

Noting that $M_{N 0}^{h} \subset M_{N}$ and $M_{T 0}^{h} \subset M_{T}$ which implies

$$
b\left(\boldsymbol{\lambda}-\boldsymbol{\lambda}^{h}, \mathbf{u}\right) \leq 0 .
$$


Using inequalities (22), (27), (28), (29) and (30) we have

$$
\begin{aligned}
\alpha\left\|\mathbf{u}^{h}-\mathbf{u}\right\|^{2}+\gamma\left\|\boldsymbol{\lambda}^{h}-\boldsymbol{\lambda}^{H}\right\|_{0, \Gamma_{C}}^{2} \leq & M\left\|\mathbf{u}^{h}-\mathbf{u}\right\|\left\|\mathbf{v}^{h}-\mathbf{u}\right\|+\left\|\lambda_{n}^{h}-\lambda_{n}\right\|_{\mathbf{W}_{N}^{\prime}}\left\|\mathbf{u}-\mathbf{v}^{h}\right\| \\
& +\left\|\lambda_{t}^{h}-\lambda_{t}\right\|_{\mathbf{W}_{T}^{\prime}}\left\|\mathbf{u}-\mathbf{v}^{h}\right\| \\
& +\left\|P_{W^{H}}\left(\lambda_{n}\right)-\lambda_{n}\right\|_{W_{N}^{\prime}}\left\|\mathbf{u}^{h}-\mathbf{u}\right\| \\
& +\left\|P_{W^{H}}\left(\lambda_{t}\right)-\lambda_{t}\right\|_{W_{T}^{\prime}}\left\|\mathbf{u}^{h}-\mathbf{u}\right\| \\
& +\left\|P_{W^{H}}\left(\lambda_{n}\right)-\lambda_{n}\right\|_{0, \Gamma_{C}}\left\|\llbracket u_{n} \rrbracket-P_{W^{H}}\left(\llbracket u_{n} \rrbracket\right)\right\|_{0, \Gamma_{C}} \\
& +\left\|P_{W^{H}}\left(\lambda_{t}\right)-\lambda_{t}\right\|_{0, \Gamma_{C}}\left\|\llbracket u_{t} \rrbracket-P_{W^{H}}\left(\llbracket u_{t} \rrbracket\right)\right\|_{0, \Gamma_{C}} .
\end{aligned}
$$

By combining inequalities (23) and (31) one obtains for all $\overline{\boldsymbol{\mu}}^{h} \in \mathbf{W}^{h}, \overline{\boldsymbol{\mu}}^{H} \in \mathbf{W}^{H}$ and $\mathbf{v}^{h} \in \mathbf{V}^{h}$

$$
\begin{aligned}
& \left(\alpha-8 M^{2} \delta\right)\left\|\mathbf{u}-\mathbf{u}^{h}\right\|^{2}+\delta \beta^{* 2}\left\|\boldsymbol{\lambda}-\boldsymbol{\lambda}^{H}\right\|_{\mathbf{W}^{\prime}}^{2}+\left(\gamma c_{1}-8 \delta\right)\left\|\boldsymbol{\lambda}^{h}-\boldsymbol{\lambda}^{H}\right\|_{\mathbf{W}^{\prime}}^{2} \\
\leq & M\left\|\mathbf{u}^{h}-\mathbf{u}\right\|\left\|\mathbf{v}^{h}-\mathbf{u}\right\|+\left\|P_{W^{H}}\left(\lambda_{n}\right)-\lambda_{n}\right\|_{W_{N}^{\prime}}\left\|\mathbf{u}^{h}-\mathbf{u}\right\|+\left\|P_{W^{H}}\left(\lambda_{t}\right)-\lambda_{t}\right\|_{W_{T}^{\prime}}\left\|\mathbf{u}^{h}-\mathbf{u}\right\| \\
& +\left\|\lambda_{n}^{h}-\lambda_{n}\right\|_{W_{N}^{\prime}}\left\|\mathbf{u}-\mathbf{v}^{h}\right\|+\left\|\lambda_{t}^{h}-\lambda_{t}\right\|_{W_{T}^{\prime}}\left\|\mathbf{u}-\mathbf{v}^{h}\right\|+\left\|P_{W^{H}}\left(\lambda_{n}\right)-\lambda_{n}\right\|_{0, \Gamma_{C}}\left\|\llbracket u_{n} \rrbracket-P_{W^{H}}\left(\llbracket u_{n} \rrbracket\right)\right\|_{0, \Gamma_{C}} \\
& +\left\|P_{W^{H}}\left(\lambda_{t}\right)-\lambda_{t}\right\|_{0, \Gamma_{C}}\left\|\llbracket u_{t} \rrbracket-P_{W^{H}}\left(\llbracket u_{t} \rrbracket\right)\right\|_{0, \Gamma_{C}}+8 \delta \beta^{* 2}\left\|\boldsymbol{\lambda}-\overline{\boldsymbol{\mu}}^{H}\right\|_{\mathbf{W}^{\prime}}^{2} \\
& +8 \delta\left\|\boldsymbol{\lambda}-\overline{\boldsymbol{\mu}}^{h}\right\|_{\mathbf{W}^{\prime}}^{2}+8 \delta\left\|\overline{\boldsymbol{\mu}}^{h}-\overline{\boldsymbol{\mu}}^{H}\right\|_{\mathbf{W}^{\prime}}^{2}, \\
\leq & \frac{\delta}{2} M^{2}\left\|\mathbf{u}-\mathbf{u}^{h}\right\|^{2}+\frac{1}{2 \delta}\left\|\mathbf{u}-\mathbf{v}^{h}\right\|^{2}+\frac{\delta}{2}\left\|\mathbf{u}-\mathbf{u}^{h}\right\|^{2}+\frac{1}{\delta}\left\|\boldsymbol{\lambda}-P_{\mathbf{W}^{H}}(\boldsymbol{\lambda})\right\|_{\mathbf{W}^{\prime}}^{2} \\
& +\frac{\xi}{2}\left\|\boldsymbol{\lambda}-\boldsymbol{\lambda}^{h}\right\|_{\mathbf{W}^{\prime}}^{2}+\frac{1}{\xi}\left\|\mathbf{u}-\mathbf{v}^{h}\right\|^{2}+\left\|P_{W^{H}}\left(\lambda_{n}\right)-\lambda_{n}\right\|_{0, \Gamma_{C}}\left\|\llbracket u_{n} \rrbracket-P_{W^{H}}\left(\llbracket u_{n} \rrbracket\right)\right\|_{0, \Gamma_{C}} \\
& +\left\|P_{W^{H}}\left(\lambda_{t}\right)-\lambda_{t}\right\|_{0, \Gamma_{C}}\left\|\llbracket u_{t} \rrbracket-P_{W^{H}}\left(\llbracket u_{t} \rrbracket\right)\right\|_{0, \Gamma_{C}} \\
& +8 \delta \beta^{* 2}\left\|\boldsymbol{\lambda}-\overline{\boldsymbol{\mu}}^{H}\right\|_{\mathbf{W}^{\prime}}^{2}+8 \delta\left\|\boldsymbol{\lambda}-\overline{\boldsymbol{\mu}}^{h}\right\|_{\mathbf{W}^{\prime}}^{2}+8 \delta\left\|\overline{\boldsymbol{\mu}}^{h}-\overline{\boldsymbol{\mu}}^{H}\right\|_{\mathbf{W}^{\prime}}^{2} .
\end{aligned}
$$

Then, for all $\overline{\boldsymbol{\mu}}^{h} \in \mathbf{W}^{h}, \overline{\boldsymbol{\mu}}^{H} \in \mathbf{W}^{H}$ and $\mathbf{v}^{h} \in \mathbf{V}^{h}$, we deduce

$$
\begin{aligned}
& \left(\alpha-\delta \frac{17 M^{2}+1}{2}\right)\left\|\mathbf{u}-\mathbf{u}^{h}\right\|^{2}+\left(\gamma c_{1}-8 \delta-\frac{\xi}{2}\right)\left\|\boldsymbol{\lambda}^{h}-\boldsymbol{\lambda}^{H}\right\|_{\mathbf{W}^{\prime}}^{2}+\left(\delta \beta^{* 2}-\frac{\xi}{2}\right)\left\|\boldsymbol{\lambda}-\boldsymbol{\lambda}^{H}\right\|_{\mathbf{W}^{\prime}}^{2} \\
\leq & \left(\frac{1}{2 \delta}+\frac{1}{\xi}\right)\left\|\mathbf{u}-\mathbf{v}^{h}\right\|^{2}+\frac{1}{\delta}\left\|P_{\mathbf{W}^{H}}(\boldsymbol{\lambda})-\boldsymbol{\lambda}\right\|_{\mathbf{W}^{\prime}}^{2}+8 \delta \beta^{* 2}\left\|\boldsymbol{\lambda}-\overline{\boldsymbol{\mu}}^{H}\right\|_{\mathbf{W}^{\prime}}^{2} \\
& +\sqrt{2}\left\|P_{\mathbf{W}^{H}}(\boldsymbol{\lambda})-\boldsymbol{\lambda}\right\|_{0, \Gamma_{C}}\left\|\llbracket \mathbf{u} \rrbracket-P_{\mathbf{W}^{H}}(\llbracket \mathbf{u} \rrbracket)\right\|_{0, \Gamma_{C}}+8 \delta\left\|\boldsymbol{\lambda}-\overline{\boldsymbol{\mu}}^{h}\right\|_{\mathbf{W}^{\prime}}^{2}+8 \delta\left\|\overline{\boldsymbol{\mu}}^{h}-\overline{\boldsymbol{\mu}}^{H}\right\|_{\mathbf{W}^{\prime}}^{2} .
\end{aligned}
$$

We recall the following standard finite-element estimates:

$$
\begin{aligned}
\left\|\mathbf{u}-\Pi^{h} \mathbf{u}\right\| & \leq C h\left\|\mathbf{u}_{r}\right\|_{2, \Omega} \\
\left\|\mathbf{u}-P_{\mathbf{W}^{H}}(\mathbf{u})\right\|_{0, \Gamma_{C}} & \leq C H^{1-\eta}\|\mathbf{u}\|_{1-\eta, \Gamma_{C}} \\
\left\|\boldsymbol{\lambda}-P_{\mathbf{W}^{h}}(\boldsymbol{\lambda})\right\|_{\mathbf{W}^{\prime}} & \leq C h\|\boldsymbol{\lambda}\|_{1 / 2, \Gamma_{C}} \\
\left\|\boldsymbol{\lambda}-P_{\mathbf{W}^{H}}(\boldsymbol{\lambda})\right\|_{\mathbf{W}^{\prime}} & \leq C H\|\boldsymbol{\lambda}\|_{1 / 2, \Gamma_{C}}
\end{aligned}
$$

Finally, the theorem is established by taking $\delta<\min \left(\frac{2 \alpha}{17 M^{2}+1} ; \frac{\gamma c_{1}}{8}\right), \xi<\min \left(2 \delta \beta^{* 2} ; 2\left(\gamma c_{1}-\right.\right.$ $8 \delta)), \mathbf{v}^{h}=\Pi^{h} \mathbf{u}$ and $\overline{\boldsymbol{\mu}}^{h}=P_{\mathbf{W}^{h}}(\boldsymbol{\lambda})$.

\subsubsection{Conforming piecewise continuous discretization for multiplier $M_{N}^{h}=M_{N 1}^{h}$ and} $M_{T}^{h}(s)=M_{T 1}^{h}(s)$

Now, we focus on the case of non-positive continuous piecewise affine multipliers where $M_{N}^{h}$ is given by (13) and $M_{T}^{h}(s)$ is given by (14) 
Theorem 3.5. Let $(\mathbf{u}, \boldsymbol{\lambda})$ be the solution to Problem (7). Assume that $\mathbf{u}_{r} \in\left(H^{2}(\Omega)\right)^{2}$ and $\boldsymbol{\lambda} \in\left(H^{1 / 2}\left(\Gamma_{C}\right)\right)^{2}$. Let $\left(\mathbf{u}^{h}, \boldsymbol{\lambda}^{h}\right)$ be the solution to the discrete problem (18) where $M_{N}^{h}=M_{N 1}^{h}$ and $M_{T}^{h}=M_{T 1}^{h}$. Then, for any $\eta>0$ we have

$$
\left\|\mathbf{u}-\mathbf{u}^{h}\right\|+\left\|\boldsymbol{\lambda}-\boldsymbol{\lambda}^{h}\right\|_{\mathbf{W}^{\prime}} \lesssim h\left\|\mathbf{u}-\chi \mathbf{u}_{s}\right\|_{2, \Omega}+H^{\frac{1}{4}}\left(\|\mathbf{u}\|_{3 / 2-\eta, \Omega}+\|\boldsymbol{\lambda}\|_{1 / 2, \Gamma_{C}}\right) .
$$

Proof. We choose $\boldsymbol{\mu}^{h}=\mathbf{r}^{h}(\boldsymbol{\lambda})=\left(r^{h} \lambda_{n}, r^{h} \lambda_{t}\right)$ in $(22)$ where $r^{h}: L^{1}\left(\Gamma_{C}\right) \mapsto W_{1}^{h}$ is a quasiinterpolation operator which preserves the non-positivity defined for any function $v$ in $L^{1}\left(\Gamma_{C}\right)$ by

$$
r^{h} v=\sum_{\mathbf{x} \in N^{h}} \alpha_{\mathbf{x}}(v) \psi_{\mathbf{x}}
$$

where $N^{h}$ represents the set of nodes $\mathbf{x}_{0}, \ldots, \mathbf{x}_{N}$ in $\overline{\Gamma_{C}}, \psi_{\mathbf{x}}$ is the scalar basis function of $W_{1}^{h}$ (defined on $\overline{\Gamma_{C}}$ ) at node $\mathbf{x}$ satisfying $\psi_{\mathbf{x}}\left(\mathbf{x}^{\prime}\right)=\delta_{\mathbf{x}, \mathbf{x}^{\prime}}$ for all $\mathbf{x}^{\prime} \in N^{h}$ and

$$
\alpha_{\mathbf{x}}(v)=\left(\int_{\Gamma_{C}} v \psi_{\mathbf{x}} d \Gamma\right)\left(\int_{\Gamma_{C}} \psi_{\mathbf{x}} d \Gamma\right)^{-1} .
$$

The approximation properties of $r^{h}$ are proved in [22]. We simply recall hereafter the two main results. The first result is concerned with $L^{2}$-stability property of $r^{h}$.

Lemma 3.6. For any $v \in L^{2}\left(\Gamma_{C}\right)$ and any $E \in T^{h}$ we have

$$
\begin{aligned}
& \left\|r^{h} v\right\|_{0, E} \lesssim\|v\|_{0, \gamma_{E}}, \\
& \text { if }|v| \leq s \text { then }\left|r^{h} v\right| \leq \text { s on } \Gamma_{C},
\end{aligned}
$$

where $\gamma_{E}=\cup_{\left\{F \in T^{h}: \bar{F} \cap \bar{E} \neq \emptyset\right\}} \bar{F}$.

Proof. Let $E \in T^{H}$ and $\psi_{1}, \psi_{2}$ be the classical scalar basic functions related to $E$. Using the definition of $\alpha_{\mathbf{x}}(v)$ and the Cauchy-Schwartz inequality we get:

$$
\begin{aligned}
\left\|r^{h} v\right\|_{0, E} & \leq \alpha_{1}\left\|\psi_{1}\right\|_{0, \Gamma_{C}}+\alpha_{2}\left\|\psi_{2}\right\|_{0, \Gamma_{C}} \\
& \leq\|v\|_{0, \gamma_{E}} \frac{\left\|\psi_{1}\right\|_{0, \Gamma_{C}}^{2}}{\int_{\Gamma_{C}} \psi_{1} d \Gamma}+\|v\|_{0, \gamma_{E}} \frac{\left\|\psi_{2}\right\|_{0, \Gamma_{C}}^{2}}{\int_{\Gamma_{C}} \psi_{2} d \Gamma} \\
& \lesssim\|v\|_{0, \gamma_{E}} .
\end{aligned}
$$

Using the definition of $\alpha_{\mathbf{x}}(\mathbf{v})$ and the partition of unity we have the second inequality:

$$
\left|r^{h} v\right|=\left|\sum_{\mathbf{x} \in N^{h}} \alpha_{\mathbf{x}}(v) \psi_{\mathbf{x}}\right| \leq \sum_{\mathbf{x} \in N^{h}}\left|\alpha_{\mathbf{x}}(v)\right| \psi_{\mathbf{x}} \leq s .
$$

Note that the proof of the first inequality of this lemma is also given in [22] using the additional assumption that the mesh $T^{h}$ is quasi-uniform. The second result is concerned with the $L^{2}$ approximation properties of $r^{h}$.

Lemma 3.7. For any $v \in H^{\eta}\left(\Gamma_{C}\right), 0 \leq \eta \leq 1$, and any $E \in T^{h}$ we have

$$
\left\|v-r^{h} v\right\|_{0, E} \lesssim h^{\eta}\|v\|_{\eta, \gamma_{E}}
$$

where $\gamma_{E}=\cup_{\left\{F \in E_{C}^{H}: \bar{F} \cap \bar{E} \neq \emptyset\right\}} \bar{F}$. 
Noting that $\lambda_{n} \leq 0$ on $\Gamma_{C}$ (resp. $\left|\lambda_{t}\right| \leq s$ on $\Gamma_{C}$ ) then $r^{h} \lambda_{n} \leq 0$ on $\Gamma_{C}$ (resp. $\left|r^{h} \lambda_{t}\right| \leq s$ on $\Gamma_{C}$ ) which implies $r^{h} \lambda_{n} \in M_{N 1}^{h}$ (resp. $r^{h} \lambda_{t} \in M_{T 1}^{h}(s)$ ). Using the inequality coming from (18) we have

$$
\begin{aligned}
b\left(\boldsymbol{\lambda}^{h}-\boldsymbol{\mu}^{h}, \mathbf{u}^{h}\right) & \leq \gamma \int_{\Gamma_{c}}\left(\boldsymbol{\lambda}^{h}-\boldsymbol{\lambda}^{H}\right)\left(\left(\boldsymbol{\mu}^{h}-\boldsymbol{\lambda}^{h}\right)-\left(\boldsymbol{\mu}^{H}-\boldsymbol{\lambda}^{H}\right)\right) d \Gamma \\
& =-\gamma\left\|\boldsymbol{\lambda}^{h}-\boldsymbol{\lambda}^{H}\right\|_{0, \Gamma_{C}}^{2}+\gamma \int_{\Gamma_{c}}\left(\boldsymbol{\lambda}^{h}-\boldsymbol{\lambda}^{H}\right)\left(\boldsymbol{\mu}^{h}-\boldsymbol{\mu}^{H}\right) d \Gamma \\
& \leq-\frac{\gamma}{2}\left\|\boldsymbol{\lambda}^{h}-\boldsymbol{\lambda}^{H}\right\|_{0, \Gamma_{C}}^{2}+\frac{\gamma}{2}\left\|\boldsymbol{\mu}^{h}-\boldsymbol{\mu}^{H}\right\|_{0, \Gamma_{C}}^{2} .
\end{aligned}
$$

Moreover

$$
\begin{aligned}
b\left(\boldsymbol{\mu}^{h}-\boldsymbol{\lambda}, \mathbf{u}^{h}-\mathbf{u}\right) & =b\left(\mathbf{r}^{h}(\boldsymbol{\lambda})-\boldsymbol{\lambda}, \mathbf{u}^{h}-\mathbf{u}\right) \\
& \leq\left\|r^{h}\left(\lambda_{n}\right)-\lambda_{n}\right\|_{0, \Gamma_{C}}\left\|\mathbf{u}^{h}-\mathbf{u}\right\|+\left\|r^{h}\left(\lambda_{t}\right)-\lambda_{t}\right\|_{0, \Gamma_{C}}\left\|\mathbf{u}^{h}-\mathbf{u}\right\| .
\end{aligned}
$$

Then we have

$$
\begin{aligned}
b\left(\boldsymbol{\mu}^{h}-\boldsymbol{\lambda}, \mathbf{u}\right) & =\int_{\Gamma_{C}}\left(r^{h}\left(\lambda_{n}\right)-\lambda_{n}\right) \llbracket u_{n} \rrbracket d \Gamma+\int_{\Gamma_{C}}\left(r^{h}\left(\lambda_{t}\right)-\lambda_{t}\right) \llbracket u_{t} \rrbracket d \Gamma, \\
& \leq\left\|r^{h}\left(\lambda_{n}\right)-\lambda_{n}\right\|_{0, \Gamma_{C}}\left\|\llbracket u_{n} \rrbracket\right\|_{0, \Gamma_{C}}+\left\|r^{h}\left(\lambda_{t}\right)-\lambda_{t}\right\|_{0, \Gamma_{C}}\left\|\llbracket u_{t} \rrbracket\right\|_{0, \Gamma_{C}} d \Gamma \\
& \leq \sqrt{2}\left\|\mathbf{r}^{h}(\boldsymbol{\lambda})-\boldsymbol{\lambda}\right\|_{0, \Gamma_{C}}\|\llbracket \mathbf{u} \rrbracket\|_{0, \Gamma_{C}} .
\end{aligned}
$$

We have $M_{N 1}^{h} \subset M_{N}$ and $M_{T 1}^{h} \subset M_{T}$ then

$$
b\left(\boldsymbol{\lambda}-\boldsymbol{\lambda}^{h}, \mathbf{u}\right) \leq 0
$$

Using inequalities (22), (33), (34), (35) and (36) we have

$$
\begin{aligned}
\alpha\left\|\mathbf{u}^{h}-\mathbf{u}\right\|^{2}+\frac{\gamma}{2}\left\|\boldsymbol{\lambda}^{h}-\boldsymbol{\lambda}^{H}\right\|_{0, \Gamma_{C}}^{2} \leq & M\left\|\mathbf{u}^{h}-\mathbf{u}\right\|\left\|\mathbf{v}^{h}-\mathbf{u}\right\|+\left\|\lambda_{n}^{h}-\lambda_{n}\right\|_{W_{N}^{\prime}}\left\|\mathbf{u}-\mathbf{v}^{h}\right\| \\
& +\left\|\lambda_{t}^{h}-\lambda_{t}\right\|_{W_{T}^{\prime}}\left\|\mathbf{u}-\mathbf{v}^{h}\right\|+\left\|r^{h}\left(\lambda_{n}\right)-\lambda_{n}\right\|_{0, \Gamma_{C}}\left\|\mathbf{u}^{h}-\mathbf{u}\right\| \\
& +\left\|r^{h}\left(\lambda_{t}\right)-\lambda_{t}\right\|_{0, \Gamma_{C}}\left\|\mathbf{u}^{h}-\mathbf{u}\right\|+\sqrt{2}\left\|\mathbf{r}^{h}(\boldsymbol{\lambda})-\boldsymbol{\lambda}\right\|_{0, \Gamma_{C}}\|\llbracket \mathbf{u} \rrbracket\|_{0, \Gamma_{C}} \\
& +\frac{\gamma}{2}\left\|\boldsymbol{\mu}^{h}-\boldsymbol{\mu}^{H}\right\|_{0, \Gamma_{C}}^{2} .
\end{aligned}
$$

By combining inequalities (23) and (37) one obtains for all $\overline{\boldsymbol{\mu}}^{h} \in \mathbf{W}^{h}$ and $\mathbf{v}^{h} \in \mathbf{V}^{h}$

$$
\begin{aligned}
& \left(\alpha-8 M^{2} \delta\right)\left\|\mathbf{u}-\mathbf{u}^{h}\right\|^{2}+\delta \beta^{* 2}\left\|\boldsymbol{\lambda}-\boldsymbol{\lambda}^{H}\right\|_{\mathbf{W}^{\prime}}^{2}+\left(\frac{\gamma c_{1}}{2}-8 \delta\right)\left\|\boldsymbol{\lambda}^{h}-\boldsymbol{\lambda}^{H}\right\|_{\mathbf{W}^{\prime}}^{2} \\
\leq & M\left\|\mathbf{u}^{h}-\mathbf{u}\right\|\left\|\mathbf{v}^{h}-\mathbf{u}\right\|+\left\|\lambda_{n}^{h}-\lambda_{n}\right\|_{W_{N}^{\prime}}\left\|\mathbf{u}-\mathbf{v}^{h}\right\|+\left\|\lambda_{t}^{h}-\lambda_{t}\right\|_{W_{T}^{\prime}}\left\|\mathbf{u}-\mathbf{v}^{h}\right\| \\
& +\left\|r^{h}\left(\lambda_{n}\right)-\lambda_{n}\right\|_{0, \Gamma_{C}}\left\|\mathbf{u}^{h}-\mathbf{u}\right\|+\left\|r^{h}\left(\lambda_{t}\right)-\lambda_{t}\right\|_{0, \Gamma_{C}}\left\|\mathbf{u}^{h}-\mathbf{u}\right\| \\
& +\sqrt{2}\left\|\mathbf{r}^{h}(\boldsymbol{\lambda})-\boldsymbol{\lambda}\right\|_{0, \Gamma_{C}}\|\llbracket \mathbf{u} \rrbracket\|_{0, \Gamma_{C}}+8 \delta \beta^{* 2}\left\|\boldsymbol{\lambda}-\overline{\boldsymbol{\mu}}^{H}\right\|_{\mathbf{W}^{\prime}}^{2}+8 \delta\left\|\boldsymbol{\lambda}-\overline{\boldsymbol{\mu}}^{h}\right\|_{\mathbf{W}^{\prime}}^{2} \\
& +8 \delta\left\|\overline{\boldsymbol{\mu}}^{h}-\overline{\boldsymbol{\mu}}^{H}\right\|_{\mathbf{W}^{\prime}}^{2}+\frac{\gamma}{2}\left\|\boldsymbol{\mu}^{h}-\boldsymbol{\mu}^{H}\right\|_{0, \Gamma_{C}}^{2}, \\
\leq & \frac{\delta}{2} M^{2}\left\|\mathbf{u}-\mathbf{u}^{h}\right\|^{2}+\frac{1}{2 \delta}\left\|\mathbf{u}-\mathbf{v}^{h}\right\|^{2}+\frac{\delta}{2}\left\|\mathbf{u}-\mathbf{u}^{h}\right\|^{2}+\frac{1}{\delta}\left\|\mathbf{r}^{h}(\boldsymbol{\lambda})-\boldsymbol{\lambda}\right\|_{0, \Gamma_{C}}^{2}+\frac{\xi}{2}\left\|\boldsymbol{\lambda}-\boldsymbol{\lambda}^{h}\right\|_{\mathbf{W}^{\prime}}^{2} \\
& +\frac{1}{\xi}\left\|\mathbf{u}-\mathbf{v}^{h}\right\|^{2}+\sqrt{2}\left\|\mathbf{r}^{h}(\boldsymbol{\lambda})-\boldsymbol{\lambda}\right\|_{0, \Gamma_{C}}\|\llbracket \mathbf{u} \rrbracket\|_{0, \Gamma_{C}}+\frac{\gamma}{2}\left\|\boldsymbol{\mu}^{h}-\boldsymbol{\mu}^{H}\right\|_{0, \Gamma_{C}}^{2} \\
& +8 \delta \beta^{* 2}\left\|\boldsymbol{\lambda}-\overline{\boldsymbol{\mu}}^{H}\right\|_{\mathbf{W}^{\prime}}^{2}+8 \delta\left\|\boldsymbol{\lambda}-\overline{\boldsymbol{\mu}}^{h}\right\|_{\mathbf{W}^{\prime}}^{2}+8 \delta\left\|\overline{\boldsymbol{\mu}}^{h}-\overline{\boldsymbol{\mu}}^{H}\right\|_{\mathbf{W}^{\prime}}^{2} .
\end{aligned}
$$


Then, for all $\overline{\boldsymbol{\mu}}^{h} \in \mathbf{W}^{h}$ and $\mathbf{v}^{h} \in \mathbf{V}^{h}$, we deduce

$$
\begin{aligned}
& \left(\alpha-\delta \frac{17 M^{2}+1}{2}\right)\left\|\mathbf{u}-\mathbf{u}^{h}\right\|^{2}+\left(\frac{\gamma c_{1}}{2}-8 \delta-\frac{\xi}{2}\right)\left\|\boldsymbol{\lambda}^{h}-\boldsymbol{\lambda}^{H}\right\|_{\mathbf{W}^{\prime}}^{2}+\left(\delta \beta^{* 2}-\frac{\xi}{2}\right)\left\|\boldsymbol{\lambda}-\boldsymbol{\lambda}^{H}\right\|_{\mathbf{W}^{\prime}}^{2} \\
\leq & \left(\frac{1}{2 \delta}+\frac{1}{\xi}\right)\left\|\mathbf{u}-\mathbf{v}^{h}\right\|^{2}+\frac{1}{\delta}\left\|\mathbf{r}^{h}(\boldsymbol{\lambda})-\boldsymbol{\lambda}\right\|_{0, \Gamma_{C}}+\sqrt{2}\left\|\mathbf{r}^{h}(\boldsymbol{\lambda})-\boldsymbol{\lambda}\right\|_{0, \Gamma_{C}}\|\llbracket \mathbf{u} \rrbracket\|_{0, \Gamma_{C}}+\frac{\gamma}{2}\left\|\boldsymbol{\mu}^{h}-\boldsymbol{\mu}^{H}\right\|_{0, \Gamma_{C}}^{2} \\
& +8 \delta \beta^{* 2}\left\|\boldsymbol{\lambda}-\overline{\boldsymbol{\mu}}^{H}\right\|_{\mathbf{W}^{\prime}}^{2}+8 \delta\left\|\boldsymbol{\lambda}-\overline{\boldsymbol{\mu}}^{h}\right\|_{\mathbf{W}^{\prime}}^{2}+8 \delta\left\|\overline{\boldsymbol{\mu}}^{h}-\overline{\boldsymbol{\mu}}^{H}\right\|_{\mathbf{W}^{\prime}}^{2} .
\end{aligned}
$$

Finally, the theorem is established by taking $\delta<\min \left(\frac{2 \alpha}{17 M^{2}+1} ; \frac{\gamma c_{1}}{16}\right), \xi<\min \left(2 \delta \beta^{* 2} ; \gamma c_{1}-\right.$ $16 \delta), \mathbf{v}^{h}=\Pi^{h} \mathbf{u}$ and $\overline{\boldsymbol{\mu}}^{h}=P_{\mathbf{W}^{h}}(\boldsymbol{\lambda})$.

\subsubsection{Nonconforming piecewise continuous discretization for multiplier $M_{N}^{h}=M_{N 1 \text {,* }}^{h}$ and $M_{T}^{h}=M_{T 1, *}^{h}(s)$}

This choice corresponds to "weakly non-positive" continuous piecewise affine multipliers where $M_{N}^{h}=M_{N 1, *}^{h}$ is given by (15) and $M_{T}^{h}=M_{T 1, *}^{h}(s)$ is given by (16).

Theorem 3.8. Let $(\mathbf{u}, \boldsymbol{\lambda})$ be the solution to Problem (7). Assume that $\mathbf{u}_{r} \in\left(H^{2}(\Omega)\right)^{2}$ and $\boldsymbol{\lambda} \in\left(H^{1 / 2}\left(\Gamma_{C}\right)\right)^{2}$. Let $\left(\mathbf{u}^{h}, \boldsymbol{\lambda}^{H}\right)$ be the solution to the discrete problem (18) where $M_{N}^{h}=M_{N 1, *}^{h}$ and $M_{T}^{h}=M_{T 1, *}^{h}(s)$. Then, for any $\eta>0$ we have

$$
\left\|\mathbf{u}-\mathbf{u}^{h}\right\|+\left\|\boldsymbol{\lambda}-\boldsymbol{\lambda}^{h}\right\|_{\mathbf{W}^{\prime}} \lesssim h\left\|\mathbf{u}-\chi \mathbf{u}_{s}\right\|_{2, \Omega}+H^{\frac{1}{2}-\frac{\eta}{2}}\left(\|\mathbf{u}\|_{3 / 2-\eta, \Omega}+\|\boldsymbol{\lambda}\|_{1 / 2, \Gamma_{C}}\right) .
$$

Proof. In (22) we choose $\mu^{h}=P_{\mathbf{W}^{h}}(\boldsymbol{\lambda})=\left(P_{W^{h}}\left(\boldsymbol{\lambda}_{n}\right), P_{W^{h}}\left(\boldsymbol{\lambda}_{t}\right)\right)$ where $P_{W^{h}}$ denotes the $L^{2}\left(\Gamma_{C}\right)$ projection onto $W_{1}^{H}$. We recall that the operator $P_{W^{h}}$ is defined for any $v \in L^{2}\left(\Gamma_{C}\right)$ by

$$
P_{W^{h}}(v) \in W_{1}^{h}, \quad \int_{\Gamma_{C}}\left(v-P_{W^{h}}(v)\right) \mu d \Gamma=0, \quad \forall \mu \in W_{1}^{h},
$$

and satisfies the following error estimates for any $0 \leq r \leq 2$ (see [10])

$$
H^{-1 / 2}\left\|v-P_{W^{h}}(v)\right\|_{-1 / 2, \Gamma_{C}}+\left\|v-P_{W^{h}}(v)\right\|_{0, \Gamma_{C}} \leq C h^{r}\|v\|_{r, \Gamma_{C}} .
$$

Clearly, $P_{\mathbf{W}^{h}}(\boldsymbol{\lambda}) \in \mathbf{M}^{h}(s)$ and using the inequality coming from (18) we have

$$
\begin{aligned}
b\left(\boldsymbol{\lambda}^{h}-\boldsymbol{\mu}^{h}, \mathbf{u}^{h}\right) & \leq \gamma \int_{\Gamma_{c}}\left(\boldsymbol{\lambda}^{h}-\boldsymbol{\lambda}^{H}\right)\left(\left(\boldsymbol{\mu}^{h}-\boldsymbol{\lambda}^{h}\right)-\left(\boldsymbol{\mu}^{H}-\boldsymbol{\lambda}^{H}\right)\right) d \Gamma \\
& =-\gamma\left\|\boldsymbol{\lambda}^{h}-\boldsymbol{\lambda}^{H}\right\|_{0, \Gamma_{C}}^{2}+\gamma \int_{\Gamma_{c}}\left(\boldsymbol{\lambda}^{h}-\boldsymbol{\lambda}^{H}\right)\left(\boldsymbol{\mu}^{h}-\boldsymbol{\mu}^{H}\right) d \Gamma \\
& \leq-\frac{\gamma}{2}\left\|\boldsymbol{\lambda}^{h}-\boldsymbol{\lambda}^{H}\right\|_{0, \Gamma_{C}}^{2}+\frac{\gamma}{2}\left\|\boldsymbol{\mu}^{h}-\boldsymbol{\mu}^{H}\right\|_{0, \Gamma_{C}}^{2} .
\end{aligned}
$$

Moreover

$$
\begin{aligned}
b\left(\boldsymbol{\mu}^{h}-\boldsymbol{\lambda}, \mathbf{u}^{h}-\mathbf{u}\right) & =b\left(P_{\mathbf{W}^{h}}(\boldsymbol{\lambda})-\boldsymbol{\lambda}, \mathbf{u}^{h}-\mathbf{u}\right) \\
& \leq\left\|P_{W^{h}}\left(\lambda_{n}\right)-\lambda_{n}\right\|_{W_{N}^{\prime}}\left\|\mathbf{u}^{h}-\mathbf{u}\right\|+\left\|P_{W^{h}}\left(\lambda_{t}\right)-\lambda_{t}\right\|_{W_{T}^{\prime}}\left\|\mathbf{u}^{h}-\mathbf{u}\right\|
\end{aligned}
$$


and

$$
\begin{aligned}
b\left(\boldsymbol{\mu}^{h}-\boldsymbol{\lambda}, \mathbf{u}\right)= & \int_{\Gamma_{C}}\left(P_{W^{h}} \lambda_{n}-\lambda_{n}\right) \llbracket u_{n} \rrbracket d \Gamma+\int_{\Gamma_{C}}\left(P_{W^{h}} \lambda_{t}-\lambda_{t}\right) \llbracket u_{t} \rrbracket d \Gamma, \\
= & \int_{\Gamma_{C}}\left(P_{W^{h}} \lambda_{n}-\lambda_{n}\right)\left(\llbracket u_{n} \rrbracket-P_{W^{h}} \llbracket u_{n} \rrbracket\right) d \Gamma \\
& +\int_{\Gamma_{C}}\left(P_{W^{h}} \lambda_{t}-\lambda_{t}\right)\left(\llbracket u_{t} \rrbracket-P_{W^{h}} \llbracket u_{t} \rrbracket\right) d \Gamma \\
\leq & \left\|P_{W^{h}} \lambda_{n}-\lambda_{n}\right\|_{0, \Gamma_{C}}\left\|\llbracket u_{n} \rrbracket-P_{W^{h}} \llbracket u_{n} \rrbracket\right\|_{0, \Gamma_{C}} \\
& +\left\|P_{W^{h}} \lambda_{t}-\lambda_{t}\right\|_{0, \Gamma_{C}}\left\|\llbracket u_{t} \rrbracket-P_{W^{h}} \llbracket u_{t} \rrbracket\right\|_{0, \Gamma_{C}}, \\
\leq & \sqrt{2}\left\|P_{\mathbf{W}^{h}}(\boldsymbol{\lambda})-\boldsymbol{\lambda}\right\|_{0, \Gamma_{C}}\left\|\llbracket \mathbf{u} \rrbracket-P_{\mathbf{W}^{h}}(\llbracket \mathbf{u} \rrbracket)\right\|_{0, \Gamma_{C}} .
\end{aligned}
$$

We have

$$
\begin{aligned}
\int_{\Gamma_{C}}\left(\lambda_{n}-\lambda_{n}^{h}\right) \llbracket u_{n} \rrbracket d \Gamma= & -\int_{\Gamma_{C}} \lambda_{n}^{h} \llbracket u_{n} \rrbracket d \Gamma, \\
\leq & \int_{\Gamma_{C}} \lambda_{n}^{h}\left(I^{h}\left(\llbracket u_{n} \rrbracket\right)-\llbracket u_{n} \rrbracket\right) d \Gamma, \\
\leq & \int_{\Gamma_{C}}\left(\lambda_{n}^{h}-\lambda\right)\left(I^{h}\left(\llbracket u_{n} \rrbracket\right)-\llbracket u_{n} \rrbracket\right) d \Gamma+\int_{\Gamma_{C}} \lambda\left(I^{h}\left(\llbracket u_{n} \rrbracket\right)-\llbracket u_{n} \rrbracket\right) d \Gamma, \\
\leq & \left\|\lambda_{n}^{h}-\lambda_{n}\right\|_{W_{N}^{\prime}}\left\|I^{h}\left(\llbracket u_{n} \rrbracket\right)-\llbracket u_{n} \rrbracket\right\|_{W_{N}} \\
& +\left\|\lambda_{n}\right\|_{0, \Gamma_{C}}\left\|I^{h}\left(\llbracket u_{n} \rrbracket\right)-\llbracket u_{n} \rrbracket\right\|_{0, \Gamma_{C}} .
\end{aligned}
$$

$$
\begin{aligned}
\int_{\Gamma_{C}}\left(\lambda_{t}-\lambda_{t}^{h}\right) \llbracket u_{t} \rrbracket d \Gamma= & \int_{\Gamma_{C}}\left(\lambda_{t}-\lambda_{t}^{h}\right)\left(\llbracket u_{t} \rrbracket-I^{h} \llbracket u_{t} \rrbracket\right) d \Gamma+\int_{\Gamma_{C}}\left(\lambda_{t}-\lambda_{t}^{h}\right) I^{h} \llbracket u_{t} \rrbracket d \Gamma \\
& -\int_{\Gamma_{C}} \lambda_{t} \llbracket u_{t} \rrbracket d \Gamma-s \int_{\Gamma_{C}}\left|\llbracket u_{t} \rrbracket\right| d \Gamma, \\
\leq & \int_{\Gamma_{C}}\left(\lambda_{t}-\lambda_{t}^{h}\right)\left(\llbracket u_{t} \rrbracket-I^{h}\left(\llbracket u_{t} \rrbracket\right)\right) d \Gamma+\int_{\Gamma_{C}} \lambda_{t}\left(I^{h}\left(\llbracket u_{t} \rrbracket\right)-\llbracket u_{t} \rrbracket\right) d \Gamma \\
& +s \int_{\Gamma_{C}}\left(\left|I^{h}\left(\llbracket u_{t} \rrbracket\right)\right|-\left|\llbracket u_{t} \rrbracket\right|\right) d \Gamma, \\
\leq & \int_{\Gamma_{C}}\left(\lambda_{t}-\lambda_{t}^{h}\right)\left(\llbracket u_{t} \rrbracket-I^{h}\left(\llbracket u_{t} \rrbracket\right)\right) d \Gamma+\int_{\Gamma_{C}} \lambda_{t}\left(I^{h}\left(\llbracket u_{t} \rrbracket\right)-\llbracket u_{t} \rrbracket\right) d \Gamma \\
& +s \int_{\Gamma_{C}}\left|I^{h}\left(\llbracket u_{t} \rrbracket\right)-\llbracket u_{t} \rrbracket\right| d \Gamma, \\
\leq & \left\|\lambda_{t}-\lambda_{t}^{h}\right\|_{W_{T}^{\prime}}\left\|\llbracket u_{t} \rrbracket-I^{h}\left(\llbracket u_{t} \rrbracket\right)\right\|_{W_{T}} \\
& +\left\|\lambda_{t}\right\|_{0, \Gamma_{C}}\left\|\llbracket u_{t} \rrbracket-I^{h}\left(\llbracket u_{t} \rrbracket\right)\right\|_{0, \Gamma_{C}}+s\left\|\llbracket u_{t} \rrbracket-I^{h}\left(\llbracket u_{t} \rrbracket\right)\right\|_{0, \Gamma_{C}} .
\end{aligned}
$$

Using (43) and (44) we have

$$
\begin{aligned}
b\left(\boldsymbol{\lambda}-\boldsymbol{\lambda}^{h}, \mathbf{u}\right) \leq & \left\|\lambda_{n}-\lambda_{n}^{h}\right\|_{W_{N}^{\prime}}\left\|\llbracket \mathbf{u} \rrbracket-I^{h} \llbracket \mathbf{u} \rrbracket\right\|_{\mathbf{w}}+\left\|\lambda_{t}-\lambda_{t}^{h}\right\|_{W_{T}^{\prime}}\left\|\llbracket \mathbf{u} \rrbracket-I^{h} \llbracket \mathbf{u} \rrbracket\right\|_{\mathbf{W}} \\
& +\sqrt{2}\|\boldsymbol{\lambda}\|_{0, \Gamma_{C}}\left\|\llbracket \mathbf{u} \rrbracket-I^{h}(\llbracket \mathbf{u} \rrbracket)\right\|_{0, \Gamma_{C}} \\
& +s\left\|\llbracket u_{t} \rrbracket-I^{h}\left(\llbracket u_{t} \rrbracket\right)\right\|_{0, \Gamma_{C}} .
\end{aligned}
$$


Using inequalities (39), (40), (41) and (45) we have

$$
\begin{aligned}
\alpha\left\|\mathbf{u}^{h}-\mathbf{u}\right\|^{2}+\gamma\left\|\boldsymbol{\lambda}^{h}-\boldsymbol{\lambda}^{H}\right\|_{0, \Gamma_{C}}^{2} \leq & M\left\|\mathbf{u}^{h}-\mathbf{u}\right\|\left\|\mathbf{v}^{h}-\mathbf{u}\right\|+\left\|\lambda_{n}^{h}-\lambda_{n}\right\|_{W_{N}^{\prime}}\left\|\mathbf{u}-\mathbf{v}^{h}\right\| \\
& +\left\|\lambda_{t}^{h}-\lambda_{t}\right\|_{W_{T}^{\prime}}\left\|\mathbf{u}-\mathbf{v}^{h}\right\|+\left\|P_{W^{h}}\left(\lambda_{n}\right)-\lambda_{n}\right\|_{W_{N}^{\prime}}\left\|\mathbf{u}^{h}-\mathbf{u}\right\| \\
& +\left\|P_{W^{h}}\left(\lambda_{t}\right)-\lambda_{t}\right\|_{W_{T}^{\prime}}\left\|\mathbf{u}^{h}-\mathbf{u}\right\| \\
& +\sqrt{2}\left\|P_{\mathbf{W}^{h}}(\boldsymbol{\lambda})-\boldsymbol{\lambda}\right\|_{0, \Gamma_{C}}\left\|\llbracket \mathbf{u} \rrbracket-P_{\mathbf{W}^{h}}(\llbracket \mathbf{u} \rrbracket)\right\|_{0, \Gamma_{C}} \\
& +\left\|\lambda_{n}-\lambda_{n}^{h}\right\|_{W_{N}^{\prime}}\left\|\llbracket u_{n} \rrbracket-I^{h}\left(\llbracket u_{n} \rrbracket\right)\right\|_{W_{N}} \\
& +\left\|\lambda_{t}-\lambda_{t}^{h}\right\|_{W_{T}^{\prime}}\left\|\llbracket u_{t} \rrbracket-I^{h}\left(\llbracket u_{t} \rrbracket\right)\right\|_{W_{T}} \\
& +\sqrt{2}\|\boldsymbol{\lambda}\|_{0, \Gamma_{C}}\left\|\llbracket \mathbf{u} \rrbracket-I^{h}(\llbracket \mathbf{u} \rrbracket)\right\|_{0, \Gamma_{C}} \\
& +s\left\|\llbracket u_{t} \rrbracket-I^{h}\left(\llbracket u_{t} \rrbracket\right)\right\|_{0, \Gamma_{C}}+\frac{\gamma}{2}\left\|\boldsymbol{\mu}^{h}-\boldsymbol{\mu}^{H}\right\|_{0, \Gamma_{C}}^{2} .
\end{aligned}
$$

By combining inequalities (23) and (46) one obtains for all $\overline{\boldsymbol{\mu}}^{h} \in \mathbf{W}^{h}$ and $\mathbf{v}^{h} \in \mathbf{V}^{h}$

$$
\begin{aligned}
& \left(\alpha-8 M^{2} \delta\right)\left\|\mathbf{u}-\mathbf{u}^{h}\right\|^{2}+\delta \beta^{* 2}\left\|\boldsymbol{\lambda}-\boldsymbol{\lambda}^{H}\right\|_{\mathbf{W}^{\prime}}^{2}+\left(\gamma c_{1}-8 \delta\right)\left\|\boldsymbol{\lambda}^{h}-\boldsymbol{\lambda}^{H}\right\|_{\mathbf{W}^{\prime}}^{2} \\
& \leq M\left\|\mathbf{u}^{h}-\mathbf{u}\right\|\left\|\mathbf{v}^{h}-\mathbf{u}\right\|+\left\|\lambda_{n}^{h}-\lambda_{n}\right\|_{W_{N}^{\prime}}\left\|\mathbf{u}-\mathbf{v}^{h}\right\|+\left\|\lambda_{t}^{h}-\lambda_{t}\right\|_{W_{T}^{\prime}}\left\|\mathbf{u}-\mathbf{v}^{h}\right\| \\
& +\left\|P_{W^{h}}\left(\lambda_{n}\right)-\lambda_{n}\right\|_{W_{N}^{\prime}}\left\|\mathbf{u}^{h}-\mathbf{u}\right\|+\left\|P_{W^{h}}\left(\lambda_{t}\right)-\lambda_{t}\right\|_{W_{T}^{\prime}}\left\|\mathbf{u}^{h}-\mathbf{u}\right\| \\
& +\sqrt{2}\left\|P_{\mathbf{W}^{h}}(\boldsymbol{\lambda})-\boldsymbol{\lambda}\right\|_{0, \Gamma_{C}}\left\|\llbracket \mathbf{u} \rrbracket-P_{\mathbf{W}^{h}}(\llbracket \mathbf{u} \rrbracket)\right\|_{0, \Gamma_{C}}+8 \delta \beta^{* 2}\left\|\boldsymbol{\lambda}-\overline{\boldsymbol{\mu}}^{H}\right\|_{\mathbf{W}^{\prime}}^{2} \\
& +\left\|\lambda_{n}-\lambda_{n}^{h}\right\|_{W_{N}^{\prime}}\left\|\llbracket u_{n} \rrbracket-I^{h}\left(\llbracket u_{n} \rrbracket\right)\right\|_{W_{N}}+\left\|\lambda_{t}-\lambda_{t}^{h}\right\|_{W_{T}^{\prime}}\left\|\llbracket u_{t} \rrbracket-I^{h}\left(\llbracket u_{t} \rrbracket\right)\right\|_{W_{T}} \\
& +\sqrt{2}\|\boldsymbol{\lambda}\|_{0, \Gamma_{C}}\left\|\llbracket \mathbf{u} \rrbracket-I^{h}(\llbracket \mathbf{u} \rrbracket)\right\|_{0, \Gamma_{C}}+s\left\|\llbracket u_{t} \rrbracket-I^{h}\left(\llbracket u_{t} \rrbracket\right)\right\|_{0, \Gamma_{C}} \\
& +\frac{\gamma}{2}\left\|\boldsymbol{\mu}^{h}-\boldsymbol{\mu}^{H}\right\|_{0, \Gamma_{C}}^{2}+8 \delta\left\|\boldsymbol{\lambda}-\overline{\boldsymbol{\mu}}^{h}\right\|_{\mathbf{W}^{\prime}}^{2}+8 \delta\left\|\overline{\boldsymbol{\mu}}^{h}-\overline{\boldsymbol{\mu}}^{H}\right\|_{\mathbf{W}^{\prime}}^{2}, \\
& \leq \frac{\delta}{2} M^{2}\left\|\mathbf{u}-\mathbf{u}^{h}\right\|^{2}+\frac{1}{2 \delta}\left\|\mathbf{u}-\mathbf{v}^{h}\right\|^{2}+\frac{\delta}{2}\left\|\mathbf{u}-\mathbf{u}^{h}\right\|^{2}+\frac{1}{\delta}\left\|\boldsymbol{\lambda}-P_{\mathbf{W}^{h}}(\boldsymbol{\lambda})\right\|_{\mathbf{W}^{\prime}}^{2}
\end{aligned}
$$

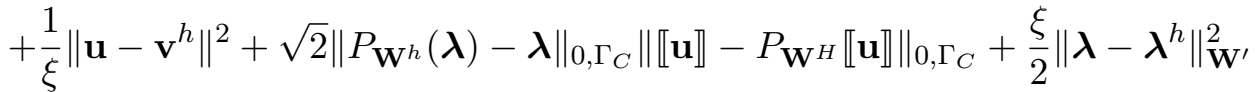

$$
\begin{aligned}
& +8 \delta \beta^{* 2}\left\|\boldsymbol{\lambda}-\overline{\boldsymbol{\mu}}^{H}\right\|_{\mathbf{W}^{\prime}}^{2}+8 \delta\left\|\boldsymbol{\lambda}-\overline{\boldsymbol{\mu}}^{h}\right\|_{\mathbf{W}^{\prime}}^{2}+8 \delta\left\|\overline{\boldsymbol{\mu}}^{h}-\overline{\boldsymbol{\mu}}^{H}\right\|_{\mathbf{W}^{\prime}}^{2} \\
& +\frac{\xi}{2}\left\|\boldsymbol{\lambda}-\boldsymbol{\lambda}^{h}\right\|_{\mathbf{W}^{\prime}}^{2}+\frac{1}{\xi}\left\|\llbracket \mathbf{u} \rrbracket-I^{h}(\llbracket \mathbf{u} \rrbracket)\right\|_{\mathbf{W}}^{2}+\sqrt{2}\|\boldsymbol{\lambda}\|_{0, \Gamma_{C}}\left\|\llbracket \mathbf{u} \rrbracket-I^{h}(\llbracket \mathbf{u} \rrbracket)\right\|_{0, \Gamma_{C}} \\
& +s\left\|\llbracket u_{t} \rrbracket-I^{h}\left(\llbracket u_{t} \rrbracket\right)\right\|_{0, \Gamma_{C}}+\frac{\gamma}{2}\left\|\boldsymbol{\mu}^{h}-\boldsymbol{\mu}^{H}\right\|_{0, \Gamma_{C}}^{2} .
\end{aligned}
$$

Then, for all $\overline{\boldsymbol{\mu}}^{h} \in \mathbf{W}^{h}$ and $\mathbf{v}^{h} \in \mathbf{V}^{h}$, we deduce

$$
\begin{aligned}
& \left(\alpha-\delta \frac{17 M^{2}+1}{2}\right)\left\|\mathbf{u}-\mathbf{u}^{h}\right\|^{2}+\left(\frac{\gamma c_{1}}{2}-8 \delta-\xi\right)\left\|\boldsymbol{\lambda}^{h}-\boldsymbol{\lambda}^{H}\right\|_{\mathbf{W}^{\prime}}^{2}+\left(\delta \beta^{* 2}-\xi\right)\left\|\boldsymbol{\lambda}-\boldsymbol{\lambda}^{H}\right\|_{\mathbf{W}^{\prime}}^{2} \\
\leq & \left(\frac{1}{2 \delta}+\frac{1}{\xi}\right)\left\|\mathbf{u}-\mathbf{v}^{h}\right\|^{2}+\frac{1}{\delta}\left\|P_{\mathbf{W}^{h}}(\boldsymbol{\lambda})-\boldsymbol{\lambda}\right\|_{0, \Gamma_{C}}+\sqrt{2}\left\|P_{\mathbf{W}^{h}}(\boldsymbol{\lambda})-\boldsymbol{\lambda}\right\|_{0, \Gamma_{C}}\left\|\llbracket \mathbf{u} \rrbracket-P_{\mathbf{W}^{H}} \llbracket \mathbf{u} \rrbracket\right\|_{0, \Gamma_{C}} \\
& +\frac{\gamma}{2}\left\|\boldsymbol{\mu}^{h}-\boldsymbol{\mu}^{H}\right\|_{0, \Gamma_{C}}^{2}+8 \delta \beta^{* 2}\left\|\boldsymbol{\lambda}-\overline{\boldsymbol{\mu}}^{H}\right\|_{\mathbf{W}^{\prime}}^{2}+8 \delta\left\|\boldsymbol{\lambda}-\overline{\boldsymbol{\mu}}^{h}\right\|_{\mathbf{W}^{\prime}}^{2}+8 \delta\left\|\overline{\boldsymbol{\mu}}^{h}-\overline{\boldsymbol{\mu}}^{H}\right\|_{\mathbf{W}^{\prime}}^{2} \\
& +\frac{1}{\xi}\left\|\llbracket \mathbf{u} \rrbracket-I^{h}(\llbracket \mathbf{u} \rrbracket)\right\|_{\mathbf{W}}^{2}+\sqrt{2}\|\boldsymbol{\lambda}\|_{0, \Gamma_{C}}\left\|\llbracket \mathbf{u} \rrbracket-I^{h}(\llbracket \mathbf{u} \rrbracket)\right\|_{0, \Gamma_{C}}+s\left\|\llbracket u_{t} \rrbracket-I^{h}\left(\llbracket u_{t} \rrbracket\right)\right\|_{0, \Gamma_{C}} .
\end{aligned}
$$

Finally, the theorem is established by taking $\delta<\min \left(\frac{2 \alpha}{17 M^{2}+1} ; \frac{\gamma c_{1}}{16}\right), \xi<\min \left(\delta \beta^{* 2} ; \frac{\gamma c_{1}}{2}-8 \delta\right)$, $\mathbf{v}^{h}=\Pi^{h} \mathbf{u}$ and $\overline{\boldsymbol{\mu}}^{h}=P_{\mathbf{W}^{h}}(\boldsymbol{\lambda})$.

Remark: Let us note that if we assume that $\mathbf{u} \in\left(H^{2}(\Omega)\right)^{2}$ in Theorem 3.8, the proved rate of convergence becomes $h^{3 / 4}$. 


\section{Numerical experiments}

The numerical tests were performed on the uncracked square defined by

$$
\bar{\Omega}=[0,1] \times[-0.5,0.5],
$$

and the considered crack is the line segment $\left.\Gamma_{C}=\right] 0,0.5[\times\{0\}$ (see Fig. 5). Three degrees of

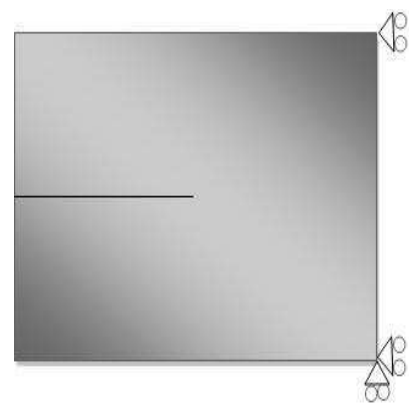

Figure 5: Cracked specimen.

freedom are blocked in order to eliminate rigid body motions (Fig. 5). In order to have both contact and non contact, slip and non slip zones between the crack lips, we impose the following body force vector field

$$
\mathbf{f}(x, y)=\left(\begin{array}{c}
0 \\
3.5 x(1-x) y \cos (2 \pi x)
\end{array}\right) .
$$

Neumann boundary conditions are prescribed as follows:

$$
\begin{aligned}
& \mathbf{g}(0, y)=\mathbf{g}(1, y)=\left(\begin{array}{c}
0 \\
0.4 \sin (2 \pi y)
\end{array}\right) \quad-0.5 \leq y \leq 0.5, \\
& \mathbf{g}(x,-0.5)=\mathbf{g}(x, 0.5)=\quad\left(\begin{array}{l}
0 \\
0
\end{array}\right) \quad 0 \leq x \leq 1 .
\end{aligned}
$$

We assume that the slip bound is constant $(s=0.09)$. An example of a unstructured mesh used is presented in Fig. 6. The numerical tests are performed with GETFEM++, the C++ finite-element library developed by Y. Renard's team (see [27]).

\subsection{Numerical solution}

The algebraic formulation of Problem (18) is given as follows

$$
\left\{\begin{array}{l}
\text { Find } U \in \mathbb{R}^{N}, L_{N} \in \bar{M}_{N}^{h} \text { and } L_{T} \in \bar{M}_{T}^{h}(s) \text { such that } \\
K U-B_{N}^{T} L_{N}-B_{T}^{T} L_{T}=F, \\
\left(\bar{L}_{N}-L_{N}\right)^{T}\left(B_{N} U+D_{N \gamma} L_{N}\right) \geq 0, \quad \forall \overline{L_{N}} \in \bar{M}_{N}^{h}, \\
\left(\bar{L}_{T}-L_{T}\right)^{T}\left(B_{T} U+D_{T \gamma} L_{T}\right) \geq 0, \quad \forall \overline{L_{T}} \in \bar{M}_{T}^{h}(s),
\end{array}\right.
$$

where $U$ is the vector of degrees of freedom (d.o.f.) for $\mathbf{u}^{h}, L_{N}$ (resp. $L_{T}$ ) is the vector of d.o.f. for the normal multiplier $\lambda_{n}^{h}$ (resp. for the tangent multiplier $\lambda_{t}^{h}$ ), $\bar{M}_{N}^{h}$ (resp. $\bar{M}_{T}^{h}(s)$ ) is the set of vectors $L_{N}$ (resp. $L_{T}$ ) such that the corresponding multiplier lies in $M_{N}^{h}$ (resp. in $M_{T}^{h}(s)$ ), $K$ is the classical stiffness matrix coming from the term $a\left(\mathbf{u}^{h}, \mathbf{v}^{h}\right), F$ is the right-hand side corresponding to the Neumann boundary condition and the volume forces, and $B_{N}, B_{T}, D_{N \gamma}$, 


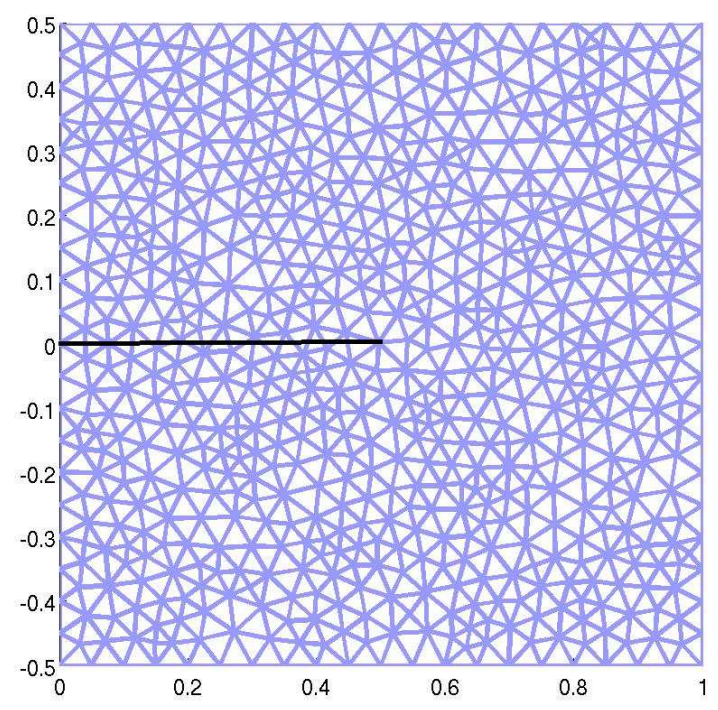

Figure 6: Unstructured mesh.

$D_{T \gamma}$ are the matrices corresponding to the terms $b\left(\lambda_{n}^{h}, \mathbf{v}^{h}\right), b\left(\lambda_{t}^{h}, \mathbf{v}^{h}\right), \gamma \int_{\Gamma_{c}}\left(\lambda_{n}^{h}-P_{W^{H}}\left(\lambda_{n}^{h}\right)\right)\left(\mu_{n}^{h}-\right.$ $\left.\lambda_{n}^{h}\right) d \Gamma$ and $\gamma \int_{\Gamma_{c}}\left(\lambda_{t}^{h}-P_{W^{H}}\left(\lambda_{t}^{h}\right)\right)\left(\mu_{t}^{h}-\lambda_{t}^{h}\right) d \Gamma$, respectively.

The inequalities in (47) can be expressed as an equivalent projection

$$
\begin{aligned}
& L_{N}=P_{\bar{M}_{N}^{h}}\left(L_{N}-r\left(B_{N} U+D_{N \gamma} L_{N}\right)\right), \\
& L_{T}=P_{\bar{M}_{T}^{h}(s)}\left(L_{T}-r\left(B_{T} U+D_{T \gamma} L_{T}\right)\right),
\end{aligned}
$$

where $r$ is a positive augmentation parameter. This last step transforms the contact conditions into nonlinear equations and we have to solve the following system:

$$
\left\{\begin{array}{l}
\text { Find } U \in \mathbb{R}^{N}, L_{N} \in \bar{M}_{N}^{h} \text { and } L_{T} \in \bar{M}_{T}^{h} \text { such that } \\
K U-B_{N}^{T} L_{N}-B_{T}^{T} L_{T}-F=0, \\
-\frac{1}{r}\left[L_{N}-P_{\bar{M}_{N}^{h}}\left(L_{N}-r\left(B_{N} U+D_{N \gamma} L_{N}\right)\right)\right]=0, \\
-\frac{1}{r}\left[L_{T}-P_{\bar{M}_{T}^{h}(s)}\left(L_{T}-r\left(B_{T} U+D_{T \gamma} L_{T}\right)\right)\right]=0 .
\end{array}\right.
$$

This allows us to use the semi-smooth Newton method (introduced for contact and friction problems in [2]) to solve Problem (50). The term 'semi-smooth' comes from the fact that projections are only piecewise differentiable. Practically, it is one of the most robust algorithms to solve contact problems with or without friction. In order to write a Newton step, one has to compute the derivative of the projection (48) and (49). An analytical expression can only be obtained when the projection itself is simple to express. This is the case for instance when the set $\mathbf{M}^{h}(s)$ is chosen such that the contact condition is satisfied on each finite-element node of the contact boundary ( $\operatorname{such}$ as $\mathbf{M}_{0}^{h}(s)$ or $\mathbf{M}_{1}^{h}(s)$ ). In this case, the projection can be expressed component-wise (see [24]). 
In order to keep the independence between the mesh and the crack, the approximation space $W^{h}$ for the multiplier is chosen to be the trace on $\Gamma_{C}$ of a Lagrange finite-element method defined on the same mesh as $\mathbf{V}^{h}$ and its degree will be specified in the following. Let us denote $X^{h}$ the space corresponding to the Lagrange finite-element method. The choice of a basis of the trace space $W^{h}=\left.X^{h}\right|_{\Gamma_{C}}$ is not completely straightforward. Indeed, the traces on $\Gamma_{C}$ of the shape functions of $X^{h}$ may be linearly dependent. A way to overcome this difficulty is to eliminate the redundant functions. Our approach in the presented numerical experiments is as follows. In a first time, we eliminate locally dependent columns of the mass matrix $\int_{\Gamma_{C}} \psi_{i} \psi_{j} d \Gamma$, where $\psi_{i}$ is the finite-element shape function of $X^{h}$, with a block-wise Gram-Schmidt algorithm. In a second time, we detect the potential remaining kernel of the mass matrix with a Lanczos algorithm.

The decomposition into patches is made using a graph partitioner algorithm. In the presented numerical tests, we use the free software METIS [23]. The nodes of the graph consist in the elements having an intersection with $\Gamma_{C}$ and the edges connect adjacent elements. Additionally, a load corresponding to the size of the intersection is considered on each elements. The partition is a very fast operation.

\subsection{Numerical tests}

In this section, we present numerical tests of the stabilized and non stabilized unilateral contact problems for the following, differently enriched, finite-element methods: $P_{2} / P_{1}, P_{2} / P_{0}, P_{1}+/ P_{1}$, $P_{1} / P_{1}, P_{1} / P_{0}$. The notation $P_{i} / P_{j}$ (resp. $P_{1}+/ P_{1}$ ) means that the displacement is approximated with a $P_{i}$ extended finite-element method (resp. a $P_{1}$ extended finite-element method with an additional cubic bubble function) and the multiplier with a continuous $P_{j}$ finite-element method for $j>0$ (resp. continuous $P_{1}$ finite-element method).

The numerical tests were performed on non-structured meshes with $h=0.108,0.057,0.03,0.016$, 0.008 , respectively. The reference solution is obtained with a structured $P_{2} / P_{1}$ method and $h=0.0021$. The von Mises stress of the reference solution is presented in Fig. 7(c). Its distribution shows that the von Mises stress is not singular at the crack lips. The normal and tangent contact stress of the reference solution are presented in Fig. 7(a) and Fig. 7(b), respectively. The normal and tangent contact stresses are not singular at the crack lips which confirms the theoretical result.

Without stabilization: The curves in the non-stabilized case are given in Fig. 8(a) for the error in the $L^{2}(\Omega)$-norm on the displacement, in Fig. 8(b) for the error in the $H^{1}(\Omega)$-norm on the displacement and in Fig. 8(c) for the error in the $L^{2}\left(\Gamma_{C}\right)$-norm on the contact stress. The $P_{1} / P_{1}$ and $P_{1} / P_{0}$ versions generally work without stabilization even though a uniform inf-sup condition cannot be proven. Fig. 8(a) shows that the rate of convergence in the error $L^{2}(\Omega)$-norm is approximatively of order 2.2 for the $P_{2} / P_{j}$ methods and of order 1.6 for the $P_{1} / P_{j}$ methods. Note that the singularity due to the transition between contact and non contact is expected to be in $H^{5 / 2-\eta}(\Omega)$ for any $\eta>0$. Theoretically, this limits the convergence rate to $3 / 2-\eta$ in the $H^{1}(\Omega)$-norm.

Fig. 8(b) shows that the rate of convergence in energy norm is approximatively of order 2 for the $P_{2} / P_{j}$ methods and of order 1.2 for the $P_{1} / P_{j}$ methods. Fig. 8(c) shows that, the rate of convergence in the error $L^{2}\left(\Gamma_{C}\right)$-norm is optimal for the $P_{2} / P_{j}$ methods (of order 1 ) and not optimal for the remaining couple of elements (approximatively of order 0.3 for the $P_{1} / P_{1}$ and $P_{1} / P_{0}$ methods and approximatively of order 0.7 for the $P_{1}+/ P_{1}$ method). It seems that the presence of some spurious modes affects these rates of convergence. 


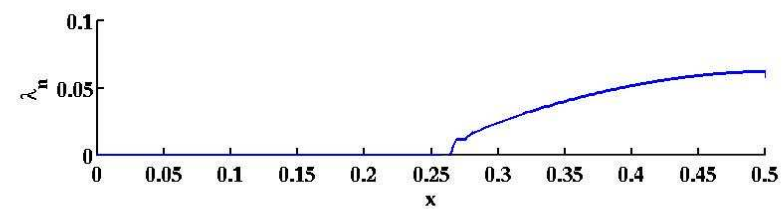

(a) Normal contact stress for the reference solution

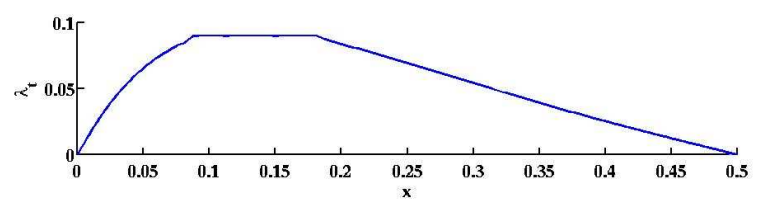

(b) Tangent contact stress for the reference solution

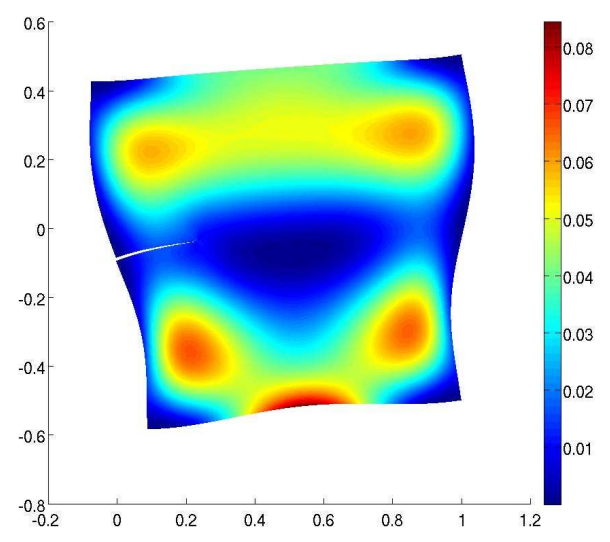

(c) Von Mises stress for the reference solution

Figure 7: Von Mises stress and contact stress for the reference solution (Note that the presence of friction in the non-contact zone (i.e. $\left.\lambda_{n} \neq 0\right)$ is due to the use of Tresca model).

Stabilized method: The curves in the stabilized case are given in Fig. 9(a) for the error in the $L^{2}(\Omega)$-norm on the displacement, in Fig. $9(\mathrm{~b})$ for the error in the $H^{1}(\Omega)$-norm on the displacement and in Fig. 9(c) for the error in the $L^{2}\left(\Gamma_{C}\right)$-norm of the contact stress. Similarly to the non stabilized method, Fig. 9(b) shows that we have an optimal rate of convergence, with a slight difference, for the error in the $L^{2}(\Omega)$-norm and the $H^{1}(\Omega)$-norm on the displacement. For the error in the $L^{2}\left(\Gamma_{C}\right)$-norm of the contact stress, Fig. $9(\mathrm{c})$ shows that the local projection stabilization eliminates the spurious modes for the $P_{1} / P_{1}, P_{1} / P_{0}$ and $P_{1}+/ P_{1}$ methods.

Concerning the error in $L^{2}\left(\Gamma_{C}\right)$-norm the value of the stabilization parameter can also be divided into two zones (see Fig. 10(a), and Fig. 10(b)). The first zone is where the error remains almost constant when the stabilization parameter $\gamma$ increases. The second zone is where the error decreases when the stabilization parameter $\gamma$ increases. Note that for a relatively large mesh size, the local projection stabilization has no influence. Now, concerning the minimal patch size, the inf-sup condition is proven to be satisfied in [16] for a size greater or equal to $3 h$. Numerically, the inf-sup condition seems to be satisfied for smaller values of the minimal patch size. In our numerical experiments, we found an optimal value between $h$ and $2 h$. For this 


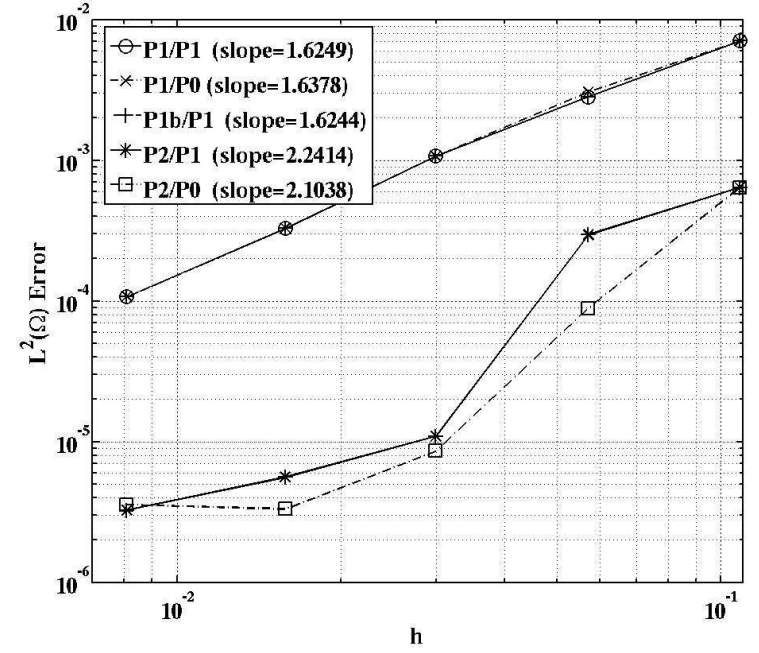

(a) Error in $L^{2}(\Omega)$-norm of the displacement

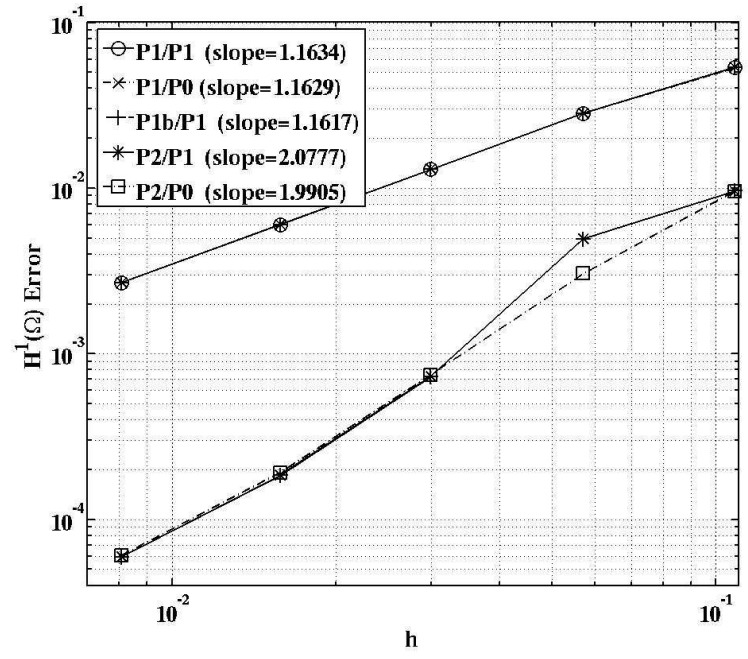

(b) Error in $H^{1}(\Omega)$-norm of the displacement

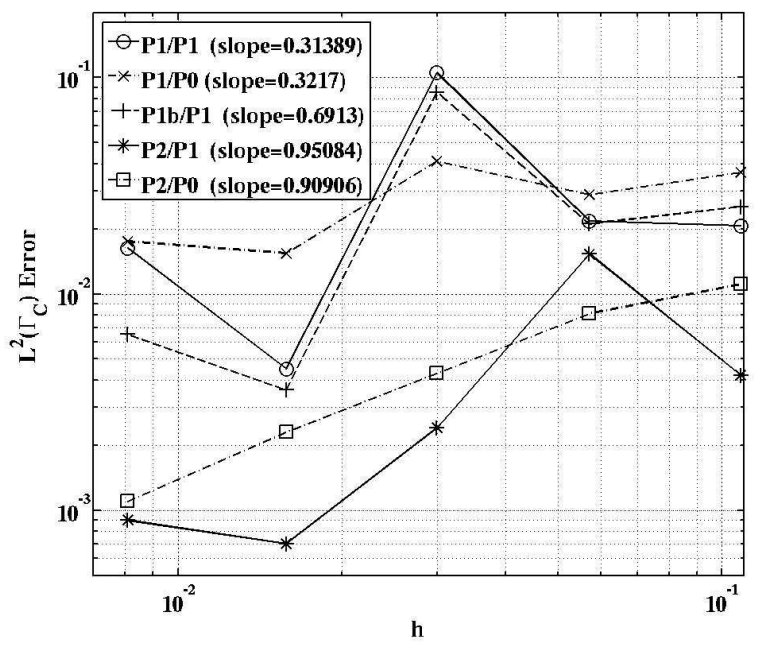

(c) Error in $L^{2}\left(\Gamma_{C}\right)$-norm of the contact stress

Figure 8: Convergence curves in the non stabilized case 


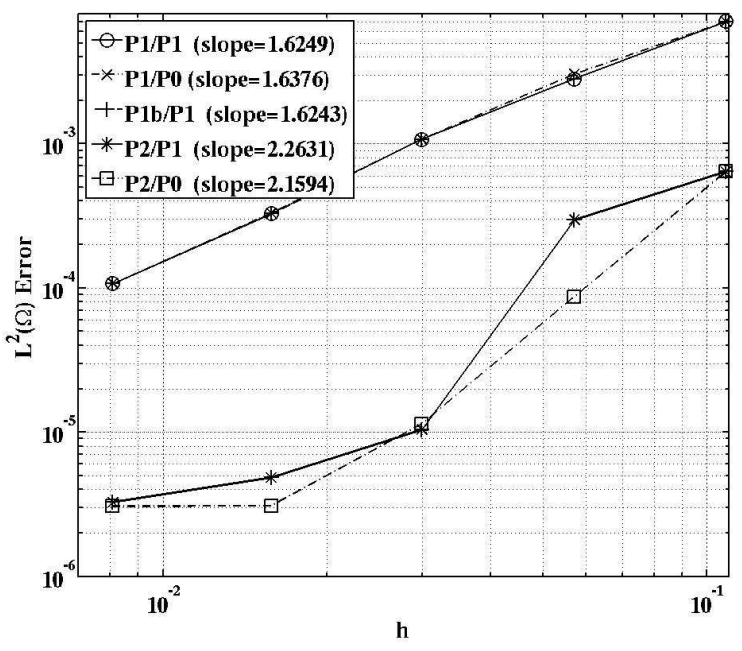

(a) Error in $L^{2}(\Omega)$-norm of the displacement

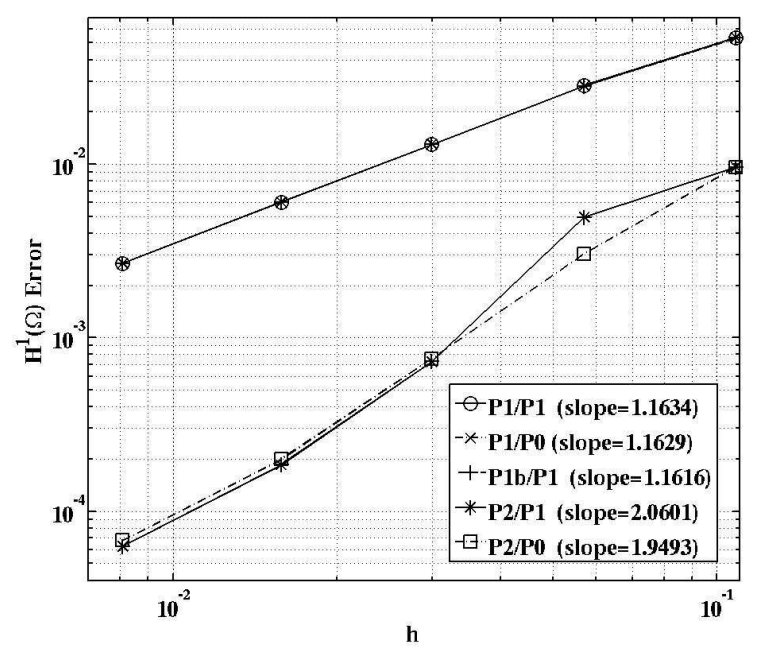

(b) Error in $H^{1}(\Omega)$-norm of the displacement

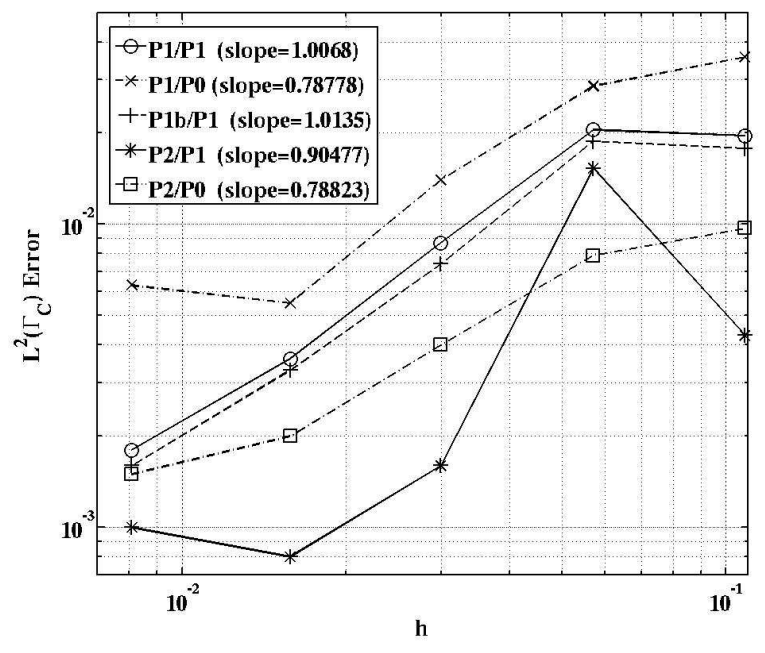

(c) Error in $L^{2}\left(\Gamma_{C}\right)$-norm of the contact stress

Figure 9: Convergence curves in the stabilized case

interval of values we have the same result, with a slight difference, as presented in Fig. 10(a) and Fig. 10(b).

\section{Conclusion}

We adapted the local projection stabilization technique to the nonlinear small strain elastostatics problem with Tresca frictional contact. A main advantage compared to some other stabilization techniques, like Barbosa-Hughes one, is that it only affects the multiplier equation in a manner that is independent of the problem to be solved.

We obtained an existence and uniqueness results for the approximated Tresca frictional contact problem in elasticity. Concerning the three contact conditions we considered theoretically, the 


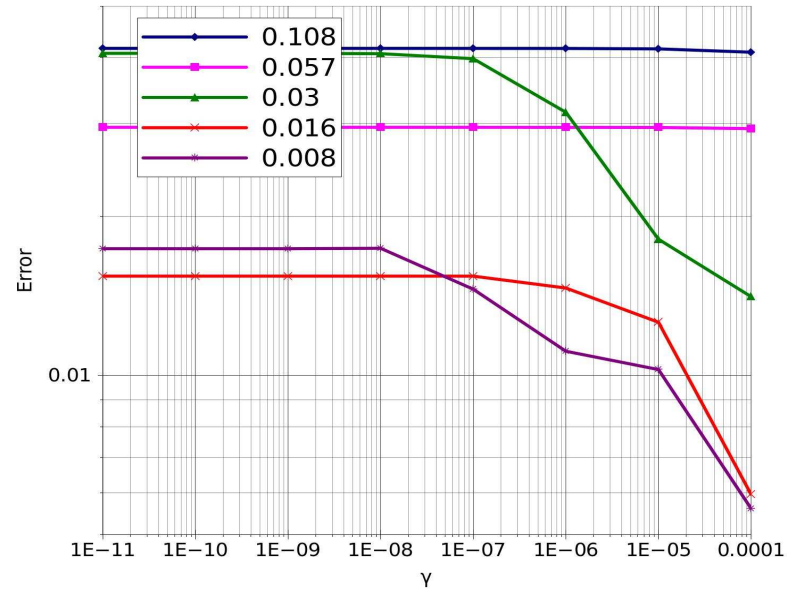

(a) $P_{1} / P_{0}$-elements

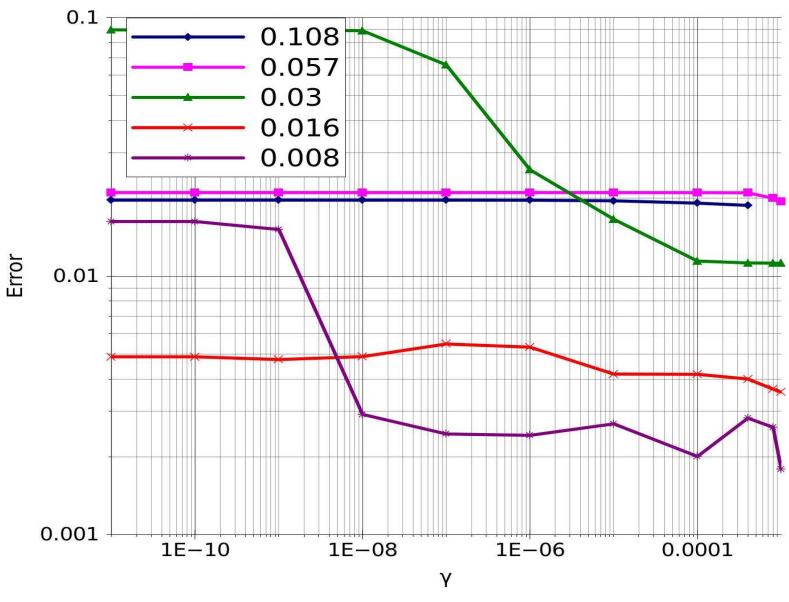

(b) $P_{1} / P_{1}$-elements

Figure 10: Influence of the stabilization parameter in $L^{2}\left(\Gamma_{C}\right)$-norm of the contact stress

given a priori error estimates are obviously sub-optimal. This is probably due to technical reasons.

In the numerical tests we considered, the stabilized methods have indeed an optimal rate of convergence. Similarly to [3], the unstabilized methods have also an optimal rate of convergence concerning the displacement. This may lead to the conclusion that no locking phenomenon was present in the numerical situation we studied despite the non-satisfaction of the discrete inf-sup condition. The fact that such a locking situation may exist or not in the studied framework (contact problem on crack lips for a linear elastic body) is an open question.

Acknowledgments. The first author acknowledges French manufacture of tires Michelin for their support.

\section{Appendix A : Proof of the Inf-sup condition}

Lemma A.1. Assume that the length of each segment $S$ of the subdivision $S^{H}$ of $\Gamma_{C}$ is more than $3 h$ and less than $L h$ with $L>0$ a given constant. Then, there exists a constant $\beta^{*}>0$, independent of $h$ and $H$, such that

$$
\forall \mu^{H} \in W^{H}, \quad \sup _{v^{h} \in V^{h}} \frac{b\left(v^{h}, \mu^{H}\right)}{\left\|v^{h}\right\|_{V}} \geq \beta^{*}\left\|\mu^{H}\right\|_{W^{\prime}} .
$$

with $b\left(v^{h}, \mu^{h}\right)=\left\langle\mu^{h}, \llbracket v^{h} \rrbracket\right\rangle_{W^{\prime}, W}$

Proof. In order to prove this condition we use a general technique introduced by Brezzi and Fortin in their book [12]. This technique can be summarized in the following proposition:

Proposition A.2 ([12]). Assume that

$$
\forall \mu \in W^{\prime}, \quad \sup _{v \in V} \frac{b(v, \mu)}{\|v\|_{V}} \geq \beta\|\mu\|_{W^{\prime}},
$$


and assume that there exists a family of uniformly continuous operators $\Pi_{h}$ from $V$ into $V^{h}$ satisfying:

$$
\begin{gathered}
b\left(\Pi_{h} w-w, \mu^{h}\right)=0, \quad \forall \mu^{h} \in\left(W^{h}\right)^{\prime}, \\
\left\|\Pi_{h} v\right\|_{V} \leqslant c\|v\|_{v},
\end{gathered}
$$

with $c$ independent of $h$. Then we have

$$
\forall \mu^{h} \in W^{h}, \quad \sup _{v^{h} \in V^{h}} \frac{b\left(v^{h}, \mu^{h}\right)}{\left\|v^{h}\right\|_{V^{h}}} \geq k_{0}\left\|\mu^{h}\right\|_{W^{\prime}},
$$

with $k_{0}=\frac{\beta}{c}$.

In our case the inf-sup condition (52) is satisfied. Indeed, in the rest of this proof, we prove the LBB condition relying on Proposition A.2.

We have the length of each segment $S$ of the subdivision $S^{H}$ of $\Gamma_{C}$ is not less than $3 h$, then similarly to [16] we can find a node $a_{S}$ such that the macro-element $\Delta_{S}$ consisting of the six triangles of $\mathcal{T}^{h}$ with common vertex $a_{S}$ satisfies the following properties:

- $S$ intersects at least one interior segment of $\Delta_{S}$ at a distance from $a_{S}$ that is no larger then half the length of this segment.

- The end point of $S$ does not belong to the interior of $\Delta_{S}$.

- If $S$ and $S^{\prime}$ are any two segments of $S^{H}, \Delta_{S} \cap \Delta_{S^{\prime}}$ is either empty or reduced to a node or a segment of $\mathcal{T}^{h}$, in other words, the macro-elements related to $S^{H}$ do not overlap.

Let $\Pi_{1}$ the $H_{1}$-stable interpolation operator of $V_{h}$ defined in [4] by:

Definition A.3. Given a displacement field $u \in H^{1}(\Omega)$ and two extensions $\tilde{u}^{1}$ and $\tilde{u}^{2}$ of $u^{1}$ and $u^{2}$ in $H^{1}(\bar{\Omega})$, respectively, we define $\Pi_{1} u$ as the element of $V^{h}$ such that:

$$
\Pi_{1} u=\sum_{j \in I \backslash I_{H}} \alpha_{j} \varphi_{j}+\sum_{j \in I_{H}}\left[\beta_{j} \varphi_{j} H_{1}+\gamma_{i} \varphi_{j} H_{2}\right]
$$

with

$$
\begin{aligned}
H_{1}(\mathbf{x}) & =\left\{\begin{array}{ll}
1 & \text { if } \mathrm{x} \in \Omega_{1}, \\
0 & \text { if } \mathbf{x} \in \Omega_{2},
\end{array} \quad H_{2}(\mathbf{x})=1-H_{1}(\mathbf{x}),\right. \\
\alpha_{i} & =\frac{1}{\left|\Delta_{i}\right|} \int_{\Delta_{i}} \tilde{u}^{k} \mathrm{~d} x \text { if } \mathbf{x}_{\mathrm{i}} \in \Omega_{\mathrm{k}}, \quad \beta_{\mathrm{i}}=\frac{1}{\left|\Delta_{\mathrm{i}}\right|} \int_{\Delta_{\mathrm{i}}} \tilde{\mathrm{u}}^{1} \mathrm{dx}, \\
\gamma_{i} & =\frac{1}{\left|\Delta_{i}\right|} \int_{\Delta_{i}} \tilde{u}^{2} \mathrm{~d} x, S_{j}:=\bigcup\left\{S \in \mathcal{T}^{h}: \operatorname{supp}\left(\varphi_{j}\right) \cap S \neq \emptyset\right\},
\end{aligned}
$$

where $\Delta_{j}$ is the maximal ball centered at $x_{j}$ such that $\Delta_{j} \subset S_{j}$ and $\left\{\mathbf{x}_{j}\right\}_{j=1}^{J}$ are the interior nodes of mesh $\mathcal{T}^{h}$.

Then for any $v \in H^{1}(\Omega)$, we propose the following restriction $\Pi_{h} v$ :

$$
\Pi_{h} v=\Pi_{1} v+\sum_{S \in S^{H}} C_{S} \mathcal{H} \varphi_{a_{S}}
$$


where $\varphi_{a_{S}}$ denotes the basis function of $V^{h}$, with support $\Delta_{S}$, that takes the value 1 at the node $a_{S}$ and 0 at all other nodes of $\mathcal{T}^{h}, \mathcal{H}$ is the Heaviside function and each constant $C_{S}$ is chosen such that

$$
\int_{S} \llbracket \Pi_{h} v \rrbracket d \Gamma=\int_{S} \llbracket v \rrbracket d \Gamma
$$

It remains to show that such constant $C_{S}$ exists and to establish the stability inequality (53a). Using the definition of $\Pi_{h} v$ we have

$$
\int_{S} \llbracket \Pi_{1} v \rrbracket-\llbracket v \rrbracket d \Gamma+\sum_{k \in S^{H}} C_{k} \int_{S} \varphi_{a_{k}} d \Gamma=0 .
$$

Using the properties of $\Delta_{S}$ we have

$$
C_{S}=-\frac{1}{\int_{S} \varphi_{a_{S}} d \Gamma} \int_{S} \llbracket \Pi_{1} v \rrbracket-\llbracket v \rrbracket d \Gamma .
$$

To derive an upper bound for the numerator of (58), we need the next two lemmas.

Lemma A.4. [16] Let $\widehat{T}$ denotes the reference unit triangle and let $\widehat{l}$ be any straight line segment that intersects $\widehat{T}$. Then, there exist a constant $\widehat{C}$, independent of $\widehat{l}$ such that

$$
\forall \widehat{w} \in H^{1}(\widehat{T}), \quad\|\widehat{w}\|_{0, \widehat{l}} \leq \widehat{C}\|\widehat{w}\|_{1, \widehat{T}} .
$$

Lemma A.5. [16] Let $l$ be a straight line segment that intersects a non degenerate triangle $T$ and let $\widehat{l}$ be its image on the reference unit triangle $\widehat{T}$ by the affine transformation that maps $\widehat{T}$ onto $T$. Let $J_{T}$ denote the Jacobian matrix of this transformation and let $\left\|\operatorname{det}\left(J_{T}\right)\right\|$ be its Euclidean norm. Then,

$$
\frac{|l|}{|\widehat{l}|} \leq\left\|J_{T}\right\|
$$

Using these two last lemmas one can prove:

Lemma A.6. [16] We always have:

$$
\int_{S} \varphi_{a_{S}} d \Gamma \geq \frac{1}{4 \sqrt{2}} h
$$

Now let us show that the operator $\Pi_{h} v$ defined by (56) satisfies the stability estimate (53b) with a constant $C$ independent of $h$ and $H$. For any $v \in H^{1}(\Omega)$, we have

$$
\left\|\Pi_{h} v\right\|_{1, \Omega} \leq\left\|\Pi_{1} v\right\|_{1, \Omega}+\left\|\sum_{S \in S^{H}} C_{S} \mathcal{H} \varphi_{a_{S}}\right\|_{1, \Omega}
$$

As each $\varphi_{a_{S}}$ has support $\Delta_{S}$ and these supports are all disjoint, we have

$$
\left\|\sum_{S \in S^{H}} C_{S} \mathcal{H} \varphi_{a_{S}}\right\|_{1, \Omega} \leq\left(\sum_{S \in S^{H}}\left|C_{S}\right|^{2}\left\|\varphi_{a_{S}}\right\|_{1, \Delta_{S}}^{2}\right)^{1 / 2}
$$

we can easily see as in [16] that there exist, a constant $\widehat{C}_{3}$ independent of $h$ and $H$ such that

$$
\left\|\varphi_{a_{S}}\right\|_{1, \Delta_{S}} \leq \widehat{C}_{3}
$$


Next let us find a bound for $C_{S}$. Let $l_{i}$ denotes the straight line segments of $S$ and $T_{i}$ the element of $\mathcal{T}^{h}$ intersected by $l_{i}$. We denote by $E^{1}$ (resp. $E^{2}$ ) the continuous extension of $\Pi_{1} v_{\Omega_{1}}$ (resp. $\left.\Pi_{1} v_{\Omega_{2}}\right)$ defined by:

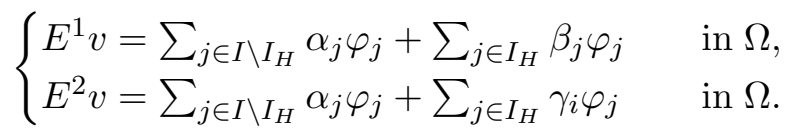

From (58) and (61), we have for all $S \in S^{H}$

$$
\begin{aligned}
\left|C_{S}\right| & \leq \frac{4 \sqrt{2}}{h}\left|\int_{S} \llbracket \Pi_{1} v \rrbracket-\llbracket v \rrbracket d \Gamma\right|, \\
& \leq \frac{4 \sqrt{2}}{h}\left(\left|\int_{S} \Pi_{1} v_{\mid \Omega_{1}}-v_{1} d \Gamma\right|+\left|\int_{S} \Pi_{1} v_{\Omega_{\Omega_{2}}}-v_{2} d \Gamma\right|\right), \\
& \leq \frac{4 \sqrt{2}}{h}\left(\sum_{i}\left|l_{i}\right|^{1 / 2}\left\|\Pi_{1} v_{\Omega_{1}}-v_{1}\right\|_{0, l_{i}}+\sum_{j}\left|l_{j}\right|^{1 / 2}\left\|\Pi_{1} v_{\Omega_{\Omega_{2}}}-v_{2}\right\|_{0, l_{j}}\right), \\
& \leq \frac{4 \sqrt{2}}{h}\left(\sum_{i}\left|l_{i}\right|^{1 / 2}\left\|\Pi_{1} v_{\mid \Omega_{1}}-\widetilde{v}_{1}\right\|_{0, l_{i}}+\sum_{j}\left|l_{j}\right|^{1 / 2}\left\|\Pi_{1} v_{\mid \Omega_{2}}-\widetilde{v}_{2}\right\|_{0, l_{j}}\right) .
\end{aligned}
$$

Then, switching to the reference element and applying Lemmas A.4 and A.5, we obtain

$$
\left|C_{S}\right| \leq \frac{4 \sqrt{2}}{h} \widehat{C}\left(\sum_{i}\left|l_{i}\right|^{1 / 2}\left\|J_{T_{i}}\right\|^{1 / 2}\left\|\widehat{E}^{1} v-\widehat{\widetilde{v}}_{1}\right\|_{1, \widehat{T}}+\sum_{j}\left|l_{j}\right|^{1 / 2}\left\|J_{T_{j}}\right\|^{1 / 2}\left\|\widehat{E}^{2} v-\widehat{\widetilde{v}}_{2}\right\|_{1, \widehat{T}}\right),
$$

where $\widehat{C}$ is the constant of Lemma A.4. Now, switching back to $T_{i}$ and $T_{j}$, we have

$$
\begin{aligned}
\left|C_{S}\right| \leq \frac{4 \sqrt{2}}{h} \widehat{C} \quad\left(\quad \sum_{i}\left|l_{i}\right|^{1 / 2}\left\|J_{T_{i}}\right\|^{1 / 2}\left|\operatorname{det} J_{T_{i}}\right|^{-1 / 2}\left(\left\|E^{1} v-\widetilde{v}_{1}\right\|_{0, T_{i}}^{2}+\left\|J_{T_{i}}\right\|^{2}\left\|E^{1} v-\widetilde{v}_{1}\right\|_{1, T_{i}}^{2}\right)^{1 / 2}\right. \\
\left.\quad+\sum_{j}\left|l_{j}\right|^{1 / 2}\left\|J_{T_{j}}\right\|^{1 / 2}\left|\operatorname{det} J_{T_{j}}\right|^{-1 / 2}\left(\left\|E_{T_{j}}^{2} v-\widetilde{v}_{2}\right\|_{0, T_{j}}+\left\|J_{T_{j}}\right\|^{2}\left\|E_{T_{j}}^{2} v-\widetilde{v}_{2}\right\|_{1, T_{j}}\right)^{1 / 2}\right) .
\end{aligned}
$$

As the triangulation $\mathcal{T}^{h}$ is trivially regular, (62) and (63) yield

$$
\left(\sum_{S \in S^{H}}\left|C_{S}\right|^{2}\left\|\varphi_{a_{S}}\right\|_{1, \Delta_{S}}^{2}\right)^{1 / 2} \leq \frac{1}{h} \widehat{C}_{4} \sqrt{L} \quad\left(\sum_{j=1}^{2}\left(\sum_{T \cap \Gamma_{c} \neq 0}\left\|E_{T}^{j} v-\widetilde{v}_{j}\right\|_{0, T}^{2}+h^{2}\left\|E_{T}^{j} v-\widetilde{v}_{j}\right\|_{1, T}^{2}\right)^{1 / 2}\right) .
$$

Using the same argument of the proof of Lemma 1 in [4] we easily show that

$$
\left(\sum_{S \in S^{H}}\left|C_{S}\right|^{2}\left\|\varphi_{a_{S}}\right\|_{1, \Delta_{S}}^{2}\right)^{1 / 2} \leq \widehat{C}_{5}\|v\|_{1, \Omega}
$$

Using this last result with Lemma 1 in [4] we have inequality (53b) which finishes the proof of Lemma A.1. 


\section{Appendix B : Singularity of the contact stress}

Lemma B.7. Assume that we have a finite number of transition points between the contact and the non contact zones and slip and non slip zones on the crack lips, then the normal and tangential contact stresses $\left(\sigma_{n}\right.$ and $\left.\sigma_{t}\right)$ are in $H^{1 / 2}\left(\Gamma_{C}\right)$.

\section{Proof.}

In order to shorten the proof we present only the analysis in the vicinity of the crack-tip. We can restrict the study to the case of a contact occurring on a neighborhood of the crack-tip, since $\sigma_{n}=0$ if there is no contact at the crack-tip.

Using the div-rot lemma, we rewrite the stress components in terms of an Airy function $\phi$ as follows:

$$
\sigma_{x x}=\frac{\partial^{2} \phi}{\partial y^{2}}, \quad \sigma_{y y}=\frac{\partial^{2} \phi}{\partial x^{2}}, \quad \sigma_{x y}=\sigma_{y x}=-\frac{\partial^{2} \phi}{\partial x \partial y} .
$$

In two-dimensional isotropic elasticity, the Hooke's law is given by:

$$
\begin{aligned}
\sigma_{x x} & =\left(\lambda_{L}+2 \mu_{L}\right) \varepsilon_{x x}+\lambda_{L} \varepsilon_{y y} \\
\sigma_{y y} & =\left(\lambda_{L}+2 \mu_{L}\right) \varepsilon_{y y}+\lambda_{L} \varepsilon_{x x} \\
\sigma_{x y} & =\mu_{L}\left(\varepsilon_{x y}+\varepsilon_{y x}\right)=2 \mu_{L} \varepsilon_{x y}
\end{aligned}
$$

So

$$
\begin{aligned}
& \varepsilon_{x y}=\varepsilon_{y x}=-\frac{1}{2 \mu_{L}} \frac{\partial^{2} \phi}{\partial x \partial y}, \\
& \varepsilon_{x x}=\frac{1}{4 \mu_{L}\left(\lambda_{L}+\mu_{L}\right)}\left(\left(\lambda_{L}+2 \mu_{L}\right) \frac{\partial^{2} \phi}{\partial y^{2}}-\lambda \frac{\partial^{2} \phi}{\partial x^{2}}\right), \\
& \varepsilon_{y y}=-\frac{1}{4 \mu\left(\lambda_{L}+\mu_{L}\right)}\left(\lambda_{L} \frac{\partial^{2} \phi}{\partial y^{2}}-\left(\lambda_{L}+2 \mu_{L}\right) \frac{\partial^{2} \phi}{\partial x^{2}}\right) .
\end{aligned}
$$

The compatibility relations

$$
\frac{\partial^{2} \varepsilon_{x x}}{\partial y^{2}}+\frac{\partial^{2} \varepsilon_{y y}}{\partial x^{2}}-2 \frac{\partial^{2} \varepsilon_{x y}}{\partial x \partial y}=0
$$

lead to the bi-harmonic equation:

$$
\frac{\lambda_{L}+2 \mu_{L}}{4 \mu_{L}\left(\lambda_{L}+\mu_{L}\right)}\left[\frac{\partial^{4} \phi}{\partial x^{4}}+\frac{\partial^{4} \phi}{\partial y^{4}}+2 \frac{\partial^{4} \phi}{\partial x^{2} \partial y^{2}}\right]=0 \quad \Longleftrightarrow \quad \Delta^{2} \phi=0,
$$

whose general solution in polar coordinates is a linear combination of the following elementary functions:

$$
r^{s+1} \cos (s-1) \theta, \quad r^{s+1} \cos (s+1) \theta, \quad r^{s+1} \sin (s-1) \theta, \quad r^{s+1} \sin (s+1) \theta .
$$

Let $\sigma_{r r}, \sigma_{\theta \theta}$ and $\sigma_{r \theta}$ be the polar stress components. By using $\mathbf{e}_{r}=(\cos \theta, \sin \theta), \mathbf{e}_{\theta}=$ $(-\sin \theta, \cos \theta)$ and the fact that $\left(\mathbf{e}_{r}, \mathbf{e}_{\theta}, \mathbf{k}\right)$ is direct and $\nabla \phi \wedge \mathbf{k}$ is independent of $x$, y, we obtain

$$
\sigma_{r r}=\frac{1}{r^{2}} \frac{\partial^{2} \phi}{\partial \theta^{2}}+\frac{1}{r} \frac{\partial \phi}{\partial r}, \quad \sigma_{\theta \theta}=\frac{\partial^{2} \phi}{\partial r^{2}}, \quad \sigma_{r \theta}=\frac{1}{r^{2}} \frac{\partial \phi}{\partial \theta}-\frac{1}{r} \frac{\partial^{2} \phi}{\partial \theta \partial r} .
$$


Besides, we have

$$
\begin{aligned}
\sigma_{x x} & =\left(\lambda_{L}+2 \mu_{L}\right) \frac{\partial u_{x}}{\partial x}+\lambda_{L} \frac{\partial u_{y}}{\partial y}, \\
\sigma_{y y} & =\left(\lambda_{L}+2 \mu_{L}\right) \frac{\partial u_{y}}{\partial y}+\lambda_{L} \frac{\partial u_{x}}{\partial x}, \\
\sigma_{x y} & =\mu_{L}\left(\varepsilon_{x y}+\varepsilon_{y x}\right)=\mu_{L}\left(\frac{\partial u_{x}}{\partial y}+\frac{\partial u_{y}}{\partial x}\right),
\end{aligned}
$$

and $\nabla \mathbf{u}=\left(\frac{\partial u_{r}}{\partial r} \mathbf{e}_{r}+\frac{\partial u_{\theta}}{\partial r} \mathbf{e}_{\theta}\right) \otimes \mathbf{e}_{r}+\left(\frac{1}{r} \frac{\partial u_{r}}{\partial \theta} \mathbf{e}_{r}+\frac{1}{r} u_{r} \mathbf{e}_{\theta}-\frac{1}{r} u_{\theta} \mathbf{e}_{r}+\frac{1}{r} \frac{\partial u_{\theta}}{\partial \theta} \mathbf{e}_{\theta}\right) \otimes \mathbf{e}_{\theta}$ where $u_{r}$ and $u_{\theta}$ are the radial and angular components of the displacement. So in polar coordinates, it becomes

$$
\begin{aligned}
\sigma_{r r} & =\left(\lambda_{L}+2 \mu_{L}\right) \frac{\partial u_{r}}{\partial r}+\frac{\lambda_{L}}{r}\left(u_{r}+\frac{\partial u_{\theta}}{\partial \theta}\right), \\
\sigma_{\theta \theta} & =\frac{\left(\lambda_{L}+2 \mu_{L}\right)}{r}\left(u_{r}+\frac{\partial u_{\theta}}{\partial \theta}\right)+\lambda_{L} \frac{\partial u_{r}}{\partial r}, \\
\sigma_{r \theta} & =\mu_{L}\left(\frac{\partial u_{\theta}}{\partial r}+\frac{1}{r} \frac{\partial u_{r}}{\partial \theta}-\frac{1}{r} u_{\theta}\right) .
\end{aligned}
$$

Consequently,

$$
\begin{aligned}
\frac{1}{r^{2}} \frac{\partial^{2} \phi}{\partial \theta^{2}}+\frac{1}{r} \frac{\partial \phi}{\partial r} & =\left(\lambda_{L}+2 \mu_{L}\right) \frac{\partial u_{r}}{\partial r}+\frac{\lambda_{L}}{r}\left(u_{r}+\frac{\partial u_{\theta}}{\partial \theta}\right), \\
\frac{\partial^{2} \phi}{\partial r^{2}} & =\frac{\left(\lambda_{L}+2 \mu_{L}\right)}{r}\left(u_{r}+\frac{\partial u_{\theta}}{\partial \theta}\right)+\lambda_{L} \frac{\partial u_{r}}{\partial r}, \\
\frac{1}{r^{2}} \frac{\partial \phi}{\partial \theta}-\frac{1}{r} \frac{\partial^{2} \phi}{\partial \theta \partial r} & =\mu_{L}\left(\frac{\partial u_{\theta}}{\partial r}+\frac{1}{r} \frac{\partial u_{r}}{\partial \theta}-\frac{1}{r} u_{\theta}\right) .
\end{aligned}
$$

In [17], Grisvard gives the corresponding displacement in polar coordinates with $\rho=1+\frac{2 \mu_{L}}{\lambda_{L}+\mu_{L}}$ :

$$
\begin{aligned}
& u_{r}=r^{s}(a \sin (s+1) \theta+b \cos (s+1) \theta+c(\rho-s) \sin (s-1) \theta-d(\rho-s) \cos (s-1) \theta), \\
& u_{\theta}=r^{s}(a \cos (s+1) \theta-b \sin (s+1) \theta-c(\rho+s) \cos (s-1) \theta-d(\rho+s) \sin (s-1) \theta),
\end{aligned}
$$

where $a, b, c, d$ are generic constants. The $P_{1}$ finite-element method will not optimally approximate the terms of this form which are not in $H^{2}(\Omega)$. So we have to determine the terms for which the real part of $s$ is such that $0<\operatorname{Re}(s)<1$.

Contact without slip: The boundary conditions for the effective contact without slip on the crack with $\theta=\pi$ can be expressed as:

$$
\begin{array}{r}
u_{\theta}(r, \pi)-u_{\theta}(r,-\pi)=0, \\
\sigma_{\theta \theta}(r, \pi)-\sigma_{\theta \theta}(r,-\pi)=0, \\
u_{r}(r, \pi)-u_{r}(r,-\pi)=0, \\
\sigma_{r \theta}(r, \pi)=\sigma_{r \theta}(r,-\pi) .
\end{array}
$$


The first equality expresses the contact condition: the jump of the normal displacement is equal to zero because we are not in the opening mode, the second equation represents the actionreaction law and the third equality expresses stick.

By using (64), these conditions read as:

$$
\begin{aligned}
u_{\theta}(r, \pi)-u_{\theta}(r,-\pi)= & 2 r^{s}(b \sin (s \pi)+d(\rho+s) \sin (s \pi)), \\
u_{r}(r, \pi)-u_{r}(r,-\pi)= & 2 r^{s}(-a \sin (s \pi)-c(\rho-s) \sin (s \pi)) \\
\sigma_{r \theta}(r, \pi)-\sigma_{r \theta}(r,-\pi)= & 4 s \mu_{L} r^{s-1}(b \sin (s \pi)+d(s-1) \sin (s \pi)) \\
\sigma_{\theta \theta}(r, \pi)-\sigma_{\theta \theta}(r,-\pi)= & r^{s-1}\left(\lambda_{L}(2 a s \sin (s+1) \pi+2 c(\rho-s) s \sin (s-1) \pi)\right. \\
& \left.+\left(\lambda_{L}+2 \mu_{L}\right)(-2 a s \sin (s+1) \pi+2 c s(\rho+s-2) \sin (s-1) \pi)\right) \\
= & 4 \mu_{L} s r^{s-1}(a \sin (s \pi)-c(s+1) \sin (s \pi)) .
\end{aligned}
$$

The determinant of the corresponding linear system can be written as:

$$
\begin{aligned}
D & =64 \mu_{L}^{2} s^{2} r^{4 s-3} \sin ^{4}(\pi s)\left|\begin{array}{cccc}
0 & 1 & -1 & 0 \\
1 & 0 & 0 & 1 \\
0 & -s-1 & -\rho+s & 0 \\
\rho+s & 0 & 0 & (s-1)
\end{array}\right| \\
& =-64 \mu_{L}^{2} s^{2} r^{4 s-3}(\rho+1)^{2} \sin ^{4}(\pi s) .
\end{aligned}
$$

So $D \neq 0$ for $0<\operatorname{Re}(s)<1$. As a consequence, there is no supplementary singular mode to the classical shear mode and the normal stress component is not singular.

Contact with slip: This case is equivalent to the result of the nonhomogeneous elastostatic problem. As the regularity of this problem is the same as the homogeneous problem (see [17]) only one singular mode is present $(s=1 / 2)$. For this singular mode, the tangent constraint is necessarily null. The boundary conditions on the crack can be expressed as:

$$
\begin{aligned}
u_{\theta}(r, \pi)-u_{\theta}(r,-\pi) & =0 \\
\sigma_{\theta \theta}(r, \pi)-\sigma_{\theta \theta}(r,-\pi) & =0 \\
\sigma_{r \theta}(r, \pi) & =0 \\
\sigma_{r \theta}(r,-\pi) & =0
\end{aligned}
$$

By using (64), these conditions read as:

$$
\begin{aligned}
u_{\theta}(r, \pi)-u_{\theta}(r,-\pi)= & 2 r^{s}(-b \sin (s+1) \pi-d(\rho+s) \sin (s-1) \pi) \\
= & 2 r^{s}(b \sin (s \pi)+d(\rho+s) \sin (s \pi)), \\
\sigma_{r \theta}(r, \pi)= & \mu_{L} r^{s-1}\left(2 a s \cos (s+1) \pi-2 b s \sin (s+1) \pi-2 c s^{2} \cos (s-1) \pi\right. \\
& \left.-2 d s^{2} \sin (s-1) \pi\right) \\
= & 2 \mu_{L} r^{s-1}\left(-a s \cos (s \pi)+b s \sin (s \pi)+c s^{2} \cos (s \pi)+d s^{2} \sin (s \pi)\right), \\
\sigma_{r \theta}(r,-\pi)= & 2 \mu_{L} r^{s-1}\left(-a s \cos (s \pi)-b s \sin (s \pi)+c s^{2} \cos (s \pi)-d s^{2} \sin (s \pi)\right), \\
\sigma_{\theta \theta}(r, \pi)-\sigma_{\theta \theta}(r,-\pi)= & r^{s-1}\left(\lambda_{L}(2 a s \sin (s+1) \pi+2 c(\rho-s) s \sin (s-1) \pi)\right. \\
& \left.+\left(\lambda_{L}+2 \mu_{L}\right)(-2 a s \sin (s+1) \pi+2 c s(\rho+s-2) \sin (s-1) \pi)\right) \\
= & r^{s-1}\left(4 \mu_{L} a s \sin (s \pi)-4 c s \mu_{L}(s+1) \sin (s \pi)\right) .
\end{aligned}
$$


which implies for $s=1 / 2$ that $a=\frac{3 c}{2}, b=0, d=0$ and $\sigma_{\theta \theta}(r, \pi)=\sigma_{\theta \theta}(r,-\pi)=0$. Then there is no supplementary singular mode to the classical shear mode and the normal stress component is not singular on the crack tip.

Let $\mathbf{m}$ be a transition point which delimits two zones of nonzero length. Using the same argument, used in the analysis on the crack tip, we show that the normal and the tangent stress component at the transition point $\mathbf{m}$ are in $H^{1 / 2}\left(\Gamma_{C}\right)$.

\section{References}

[1] R. Adams, Sobolev Spaces, Academic Press, New York, 1975.

[2] P. Alart And A. Curnier, A mixed formulation for frictional contact problems prone to Newton like solution methods, Comput. Methods Appl. Mech. Engrg., 92 (1991), pp. 353375 .

[3] S. Amdouni, P. Hild, V. Lleras, M. Moakher, and Y. Renard, A stabilized Lagrange multiplier method for the enriched finite-element approximation of contact problems of cracked elastic bodies, ESAIM Math. Model. Numer. Anal., 46 (2012), pp. 813-839.

[4] S. Amdouni, K. Mansouri, Y. Renard, M. Arfaoui, and M. Moakher, Numerical convergence and stability of mixed formulation with X-FEM cut-off, European Journal of Computational Mechanics/Revue Européenne de Mécanique Numérique, 0 (2012), pp. 1-14.

[5] S. Amdouni, M. Moakher, And Y. Renard, A local projection stabilization of fictitious domain method for elliptic boundary value problems, submitted.

[6] L. Baillet And T. Sassi, Mixed finite element methods for the Signorini problem with friction, Numer. Methods Partial Differential Equations, 22 (2006), pp. 1489-1508.

[7] H. J. Barbosa And T. Hughes, The finite element method with Lagrange multipliers on the boundary: circumventing the Babuška-Brezzi condition, Comput. Methods Appl. Mech. Engrg., 85 (1991), pp. 109-128.

[8] — Boundary Lagrange multipliers in finite element methods: error analysis in natural norms, Numer. Math., 62 (1992), pp. 1-15.

[9] G. R. Barrenechea and F. Chouly, A local projection stabilized method for fictitious domains, Applied Mathematics Letters, 25 (2012), pp. 2071 - 2076.

[10] F. Ben Belgacem and Y. Renard, Hybrid finite element methods for the Signorini problem, Math. Comp., 72 (2003), pp. 1117-1145.

[11] F. Brezzi And M. Fortin, A minimal stabilisation procedure for mixed finite element methods, Numer. Math., 89 (2001), pp. 457-491.

[12] F. Brezzi And M. Fortin, Mixed and Hybrid Finite Element Methods, vol. 15, Springer Series in Computational Mathematics, New York, 1991. 
[13] E. Burman, Projection stabilisation of Lagrange multipliers for the imposition of constraints on interfaces and boundaries, ArXiv e-prints, (2012).

[14] E. Chahine, P. Laborde, and Y. Renard, Crack-tip enrichment in the X-FEM method using a cut-off function, Int. J. Numer. Meth. Engng., 75 (2008), pp. 629-646.

[15] P. Ciarlet, The finite element method for elliptic problems, in Handbook of Numerical Analysis, Volume II, Part 1, P. Ciarlet and J. Lions, eds., North Holland, 1991, pp. 17-352.

[16] V. Girault and R. Glowinski, Error analysis of a fictitious domain method applied to a Dirichlet problem, Japan J. Indust. Appl. Math., 12 (1995), pp. 487-514.

[17] P. Grisvard, Elliptic Problems in Nonsmooth Domains, Pitman, 1985.

[18] J. Haslinger and I. Hlavávcek, Approximation of the Signorini problem with friction by a mixed finite element method, J. Math. Anal. Appl., 86 (1982), pp. 99-122.

[19] J. Haslinger, I. HlaváČEK, And J. NeČAs, Numerical methods for unilateral problems in solid mechanics, in Handbook of Numerical Analysis, Volume IV, Part 2, P. Ciarlet and J.-L. Lions, eds., North Holland, 1996, pp. 313-485.

[20] J. Haslinger and Y. Renard, A new fictitious domain approach inspired by the extended finite element method, to appear in SIAM J. Numer. Anal.

[21] J. Haslinger And T. SAssi, Mixed finite element approximation of 3D contact problems with given friction: error analysis and numerical realization, M2AN Math. Model. Numer. Anal., 38 (2004), pp. 563-578.

[22] P. Hild And Y. Renard, An error estimate for the Signorini problem with Coulomb friction approximated by finite elements, SIAM J. Numer. Anal., 45 (2007), pp. 2012-2031 (electronic).

[23] G. KARYPIS AND V. KUMAR, Metis: Unstructured graph partitioning and sparse matrix ordering system. http://www.cs.umn.edu/ metis.

[24] H. B. Khenous, J. Pommier, And Y. Renard, Hybrid discretization of the Signorini problem with Coulomb friction. Theoretical aspects and comparison of some numerical solvers, Appl. Numer. Math., 56 (2006), pp. 163-192.

[25] M. Moussaoui and K. Khodja, Regularité des solutions d'un problème mêlé DirichletSignorini dans un domaine polygonal plan, Commun. Partial Differential Equations, 17 (1992), pp. 805-826.

[26] S. Nicaise, Y. Renard, And E. Chahine, Optimal convergence analysis for the extended finite element method, Internat. J. Numer. Methods Engrg., 86 (2011), pp. 528-548.

[27] J. Pommier and Y. Renard, Getfem ++ , an open source generic $C++$ library for finite element methods. http://www-gmm.insa-toulouse.fr/getfem.

[28] T. SASsi, Conconforming mixed variational formulation for the signorini problem with a given friction, Preprint of MAPLY Laboratoire de Mathématiques Appliquées de LYon, (2003).

[29] D. Silvester, Optimal low order finite element methods for incompressible flow, Comput. Methods Appl. Mech. Engrg., 111 (1994), pp. 357-368. 